\title{
Age distribution of young clusters and field stars in the Small Magellanic Cloud $\star, \star \star$
}

\author{
E. Chiosi ${ }^{1}$, A. Vallenari ${ }^{2}$, E. V. Held ${ }^{2}$, L. Rizzi ${ }^{3}$, and A. Moretti ${ }^{1}$ \\ 1 Astronomy Department, Padova University, Vicolo dell'Osservatorio 2, 35122 Padova, Italy \\ e-mail: emanuela.chiosi@oapd.inaf.it;moretti@pd.astro.it \\ 2 INAF, Padova Observatory, Vicolo dell'Osservatorio 5, 35122 Padova, Italy \\ e-mail: [antonella.vallenari; enrico.held]@oapd.inaf.it \\ 3 Institute for Astronomy, 2680 Woodlawn Drive, Hawaii 96822-1897, USA \\ Received 21 November 2005 / Accepted 15 February 2006
}

ABSTRACT

\begin{abstract}
Aims. In this paper we discuss the cluster and field star formation in the central part of the Small Magellanic Cloud. The main goal is to study the correlation between young objects and their interstellar environment.

Methods. The ages of about 164 associations and 311 clusters younger than $1 \mathrm{Gyr}$ are determined using isochrone fitting. The spatial distribution of the clusters is compared with the HI maps, with the HI velocity dispersion field, with the location of the CO clouds and with the distribution of young field stars.

Results. The cluster age distribution supports the idea that clusters formed in the last 1 Gyr of the SMC history in a roughly continuous way with periods of enhancements. The two super-shells 37A and 304A detected in the HI distribution are clearly visible in the age distribution of the clusters: an enhancement in the cluster formation rate has taken place from the epoch of the shell formation. A tight correlation between young clusters and the $\mathrm{HI}$ intensity is found. The degree of correlation is decreasing with the age of the clusters. Clusters older than $300 \mathrm{Myr}$ are located away from the HI peaks. Clusters and associations younger than $10 \mathrm{Myr}$ are related to the CO clouds in the SW region of the SMC disk. A positive correlation between the location of the young clusters and the velocity dispersion field of the atomic gas is derived only for the shell 304A, suggesting that the cloud-cloud collision is probably not the most important mechanism of cluster formation. Evidence of gravitational triggered episode due to the most recent close interaction between SMC and LMC is found both in the cluster and field star distribution.
\end{abstract}

Key words. galaxies: Magellanic Clouds - galaxies: star clusters - galaxies: stellar content

\section{Introduction}

Star formation is a complex phenomenon involving at the same time several physical processes such as turbulence, gravitational collapse, cooling, gravitational trigger. There are many open questions about star formation in galaxies: is it a continuous process or does it proceed by bursts? Which are the fundamental triggers of star formation and in which measure are they internal or external? The Large and Small Magellanic Clouds (LMC, SMC) which are believed to be interacting with each other, are ideal laboratories to study the process of field star and cluster formation.

LMC has been widely studied using both ground based and HST data (Bertelli et al. 1992; Vallenari et al. 1996; Gallagher et al. 1996; Holtzman et al. 1997; Elson et al. 1997; Geha et al. 1998; Harris \& Zaritsky 1999, 2004; Olsen 1999; Dolphin 2000; Javiel et al. 2005). The vast majority of the authors suggest that the LMC experimented a continuous SF with several enhancements, roughly at 2-4 Gyr and at 6-7 Gyr although the precise epochs change from field to field. At the opposite the SMC has been less studied. The SMC shows an asymmetric appearance with an irregular main body and an eastern extension. In a photographic plate study Gardiner \& Hatzidimitriou (1992) found that

\footnotetext{
* Based on observations taken at ESO under proposal 66B-0615.

$\star \star$ Table 2 is only available in electronic form at http://www.edpsciences.org
}

the bulk of the stellar population in the SMC is about 10 Gyr old. They observe that the young stellar population is biased toward the eastern LMC-facing side of the SMC. Crowl et al. (2001) find the same trend among the SMC populous clusters: those toward the eastern side tend to be younger and more metal rich than those on the western side. Recently Zaritsky et al. (2000), Maragoudaki et al. (2001) and Cioni et al. (2000) confirmed that the asymmetric structure of the SMC is due exclusively to the distribution of young main sequence stars, while old stars show a rather regular and smooth distribution typical of a spheroidal body. The asymmetric distribution of the young stars is consistent with the patterns of the associations and HII regions (Bica $\&$ Schmitt 1995). This is interpreted as the effect of the perturbations developed by the interaction of LMC-SMC.

Concerning the SFR of the field stars in the SMC, no real consensus is reached whether the star formation has proceeded with several periods of enhancements, namely at $400 \mathrm{Myr}, 3 \mathrm{Gyr}$, 9 Gyr as found by Harris \& Zaritsky (2004) in the SMC disk, or in a more continuous way, with a main episode between 5 and $8 \mathrm{Gyr}$ as derived by Dolphin et al. (2001) in the halo. A large population of clusters is found in the SMC. Hodge (1986), comparing the number of clusters found down to $B=22$ (inner regions) or $B=23$ (outer regions) in selected regions with the number of known clusters in SMC catalogs at that time, estimated a global population of 900 clusters. Considering incompleteness effects, 2000 clusters are expected if small older 
clusters were detectable. Pietrzynski \& Udalski (2000) catalog includes 238 clusters down to $B \sim 21.1$ or $V \sim 21.5$. Bica \& Dutra (2000) including in the catalog faint and loose systems, find 633 clusters. Considering also the clusters related to emission (NC and $\mathrm{CN}$ types) the total number of objects is 719. As pointed out by the authors, the number of clusters in Bica \& Dutra (2000) is still far from being complete. Concerning cluster age distribution, the SMC is known to have at least six populous clusters of intermediate age in the range 5-9 Gyr, but only one true old object (NGC 121) having an age of $>10$ Gyr (Stryker et al. 1985; Dolphin et al. 2001). Only a few of the clusters in Bica \& Dutra (2000) catalog have known ages. Pietrzynski \& Udalski (1999) using isochrone fitting, and Rafelski \& Zaritsky (2005) making use of integrated colors derive the age of a limited number of bright clusters, namely 93, and 200 respectively. Piatti et al. (2005) discuss the age and metallicity for 36 SMC clusters. The data seem to suggest that the formation of young clusters took place in bursts (Rafelski \& Zaritsky 2005; Piatti et al. 2001; Rich et al. 2000).

The aim of this work is to cast light on the process of field and cluster formation in this galaxy deriving the age distribution of a large number of clusters in the central regions of the SMC and comparing it with the field star formation. At this purpose we make use of the OGLE data for the whole SMC disk, and of better quality data obtained at the ESO $2.2 \mathrm{~m}$ telescope for a region around NGC 269, located at the SE end of the disk, at the border of the supershell 37A. In Sect. 2 the observations and data reduction are described. In Sect. 3 the SMC distance, reddening, metallicity, and line of sight depth are given. In Sect. 4 the cluster age distribution in the whole disk is derived. In Sect. 5 the two supershells, 37A and 304A are discussed, in Sect. 6 the spatial distribution of the clusters is compared with the environment properties. In Sect. 7 field star formation rate is discussed. Finally, concluding remarks are given in Sect. 8.

\section{Data, observations, and reduction}

\subsection{NGC 269 region}

$V, I$ images were taken with the WFI at the ESO $2.2 \mathrm{~m}$ telescope on 2000, October 21 under photometric conditions as backup observations for a different project. The field of view is of $34 \times 33 \mathrm{arcmin}^{2}$. It is centered on the cluster NGC 269 at $\alpha=00^{\mathrm{h}} 48^{\mathrm{m}} 30.6^{\mathrm{s}}$ and $\delta=-73^{\circ} 31^{\prime} 30^{\prime \prime}$. The exposure times are $300 \mathrm{~s}$ in $V$ and $300 \mathrm{~s}$ in $I$. To avoid saturation at the bright magnitude end two images having exposure times of $20 \mathrm{~s}$ were taken. The seeing was 1 arcsec.

Pre-reduction of CCD images was performed within the IRAF environment. Each image was bias-subtracted and flatfielded using twilight sky flats. After these steps, all images were astrometrically calibrated using the IRAF package MSCRED (Valdes 1998) and the script package WFPRED developed at the Padova Observatory (Rizzi 2003). Photometry was obtained with DAOPHOT (Stetson 1987).The photometric zero points were then set by comparison with secondary standard stars accurately calibrated onto the Landolt (1992) system. The estimated uncertainty of the zero point calibration is $0.03 \mathrm{mag}$ in both $V$ and $I$. More than 100000 stars are found down to $V=24$. Figure 1 presents the CMD of the whole region. The completeness correction is calculated as usual by means of artificial stars experiments where a small number of artificial stars are injected in the original frames. Then the frames are reduced following the same procedure. The completeness factors in $V$ and $I$ band, $\Lambda_{V}$, and $\Lambda_{I}$ respectively, defined as the number of recovered

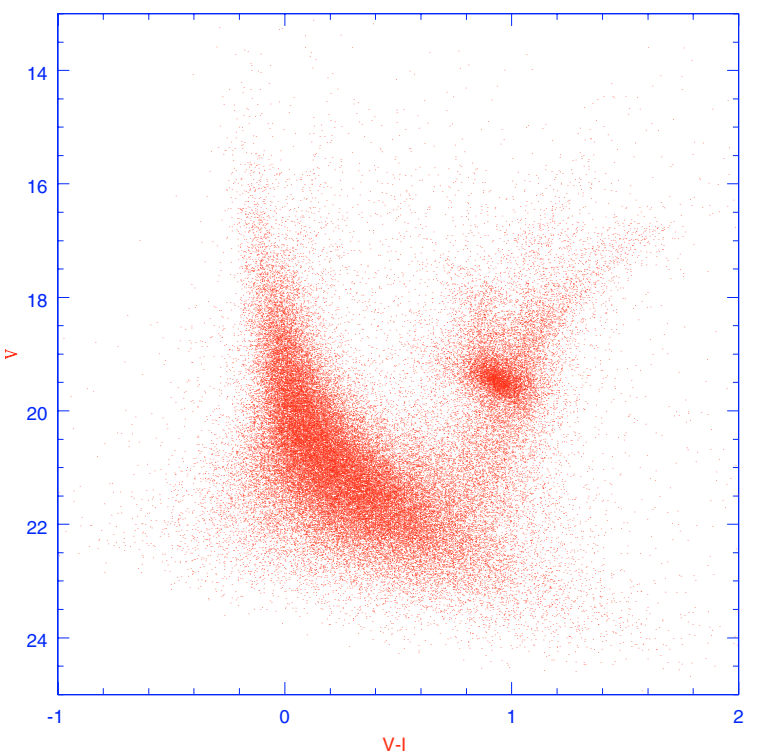

Fig. 1. The CMD of the observed region around NGC 269.

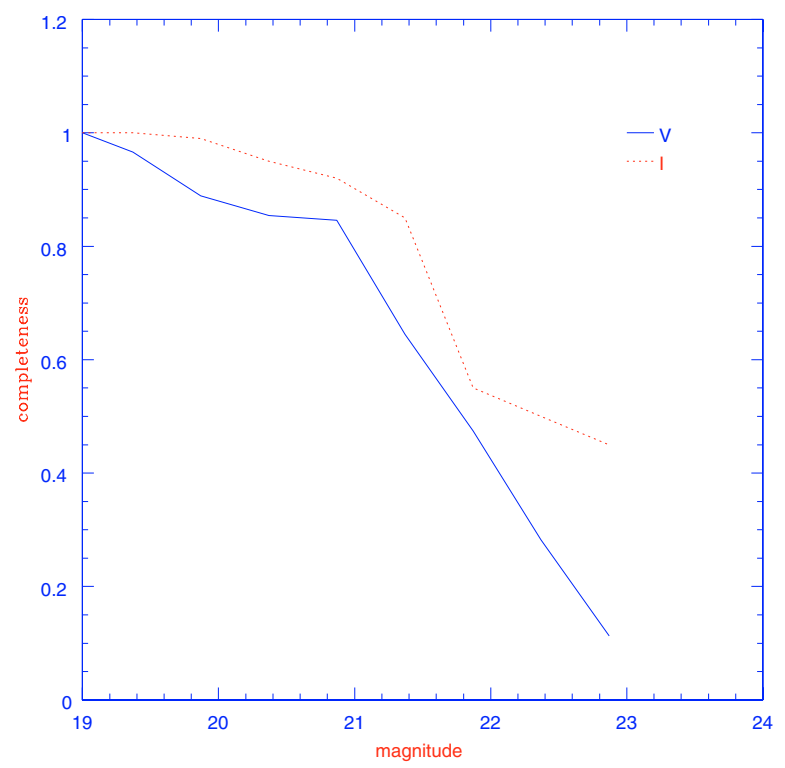

Fig. 2. Completeness factors $\Lambda$ in $V$ and $I$ magnitude are plotted as functions of the magnitude.

on the added stars are plotted in Fig. 2. The data are complete at $50 \%$ level for magnitudes brighter than $V<22$. Photometric errors are derived from artificial stars experiments and are plotted in Fig. 3.

\subsection{OGLE data}

An analysis over a wider area of the galaxy is made using Optical Gravitational Lensing Experiment (OGLE II) data (Udalski et al. 1998) to derive cluster and field star age distribution for the regions not covered by our photometry. The data cover 2.4 sq. deg. of the main body of the SMC. The limiting magnitude of the photometry is $V \sim 21.5$. Completeness correction is applied following Udalski et al. (1998). Field population data are complete at about $80 \%$ level and $89 \%$ level down to $V=20.5 \mathrm{mag}$, in the most and in the least crowded fields respectively. For magnitudes brighter than $V=20.5$, the incompleteness correction is not very significant in the field population. The $50 \%$ level is 


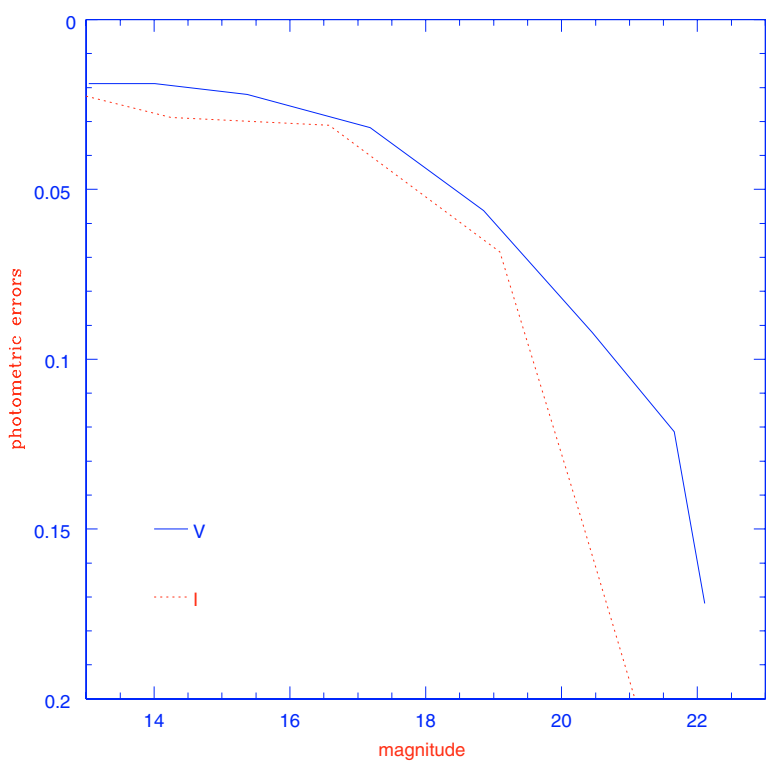

Fig. 3. Photometric errors as functions of the $V$ and $I$ magnitude.

reached at about $V=21 \mathrm{mag}$ in the most crowded fields. This mean incompleteness correction is suitable not only for the field population, but as well for sparse clusters. Inside dense clusters the completeness might be very different. In order to estimate the incompleteness correction in these objects, we compare the region of NGC 269 we observe and of which we estimate the completeness, with OGLE data. We select the core of dense clusters. The incompleteness correction we derive for the field population is in agreement with the nominal values by Udalski et al. (1998). Inside the radius of the densest clusters given by Bica \& Dutra (2000), the OGLE data are complete at $80 \%, 70 \%$ and $50 \%$ level at magnitudes $V=19.0,19.5,20.3$ respectively. This correction is then applied to derive the ages of dense clusters.

\section{SMC distance modulus, reddening, line of sight depth, metallicity}

A distance modulus of $(m-M)_{0}=18.9$ is assumed for the SMC, in agreement with recent determinations by Storm et al. (2004), Weldrake et al. (2004), Caputo et al. (1999) and Sandage et al. (1999).

The extinction map across the SMC was recently determined by Zaritsky et al. (2002) using data from the Magellanic Cloud Photometric surveys. They show that the extinction varies spatially increasing along the SMC ridge from northeast to southwest. In addition, young stars present an average differential extinction 0.3 mag higher than old stars. By comparison, a mean reddening $E(B-V)=0.08 \mathrm{mag}$ is derived by Tumlinson et al. (2002) and Hunter et al. (2003). In the following, when possible, we derive the reddening of each cluster by main sequence fitting. Otherwise, a mean value of $E(B-V)=0.08$ mag of the reddening is assumed. In the case of field star population we account for an additional reddening Gaussian dispersion with $\sigma=0.09$. No differential extinction is included.

The line of sight depth of the SMC is a long-lasting controversial issue. Mathewson et al. (1986) derive a great depth of $30 \mathrm{kpc}$ by measuring distances and radial velocities of Cepheid stars. Welch et al. (1987) concluded that the SMC does not extend beyond its tidal radius (4-9 kpc). Groenewegen (2000) using near infrared data of Cepheids derives a depth of $14 \mathrm{kpc}$. Crowl et al. (2001) using the magnitudes of the clump stars in clusters derive a depth ranging between 6 and $12 \mathrm{kpc}$. The main reasons of those discrepancies can be found in the uncertainties on the extinction and on the relation period-luminositymetallicity of Cepheid. Stanimirović et al. (2004) point out that the application of the correction for differential reddening derived by Zaritsky et al. (2002) significantly influences the distance determinations bringing the depth of the SMC within its tidal radius (4-9 kpc). Additionally, while the tidal tails contribute mostly to the elongation of the galaxy, the main body of the SMC does not present a significant elongation $(\sim 5 \mathrm{kpc})$. A recent determination is made by Lah et al. (2005) who, using the $\mathrm{P}-\mathrm{L}$ relation of OGLE II variables, find a very patchy structure with a depth of about $3.2 \pm 1.6 \mathrm{kpc}$. In the following, discussing the SFR of the field stars, we adopt an intermediate value of $4 \mathrm{kpc}$ implying a difference in the distance modulus of about $0.14 \mathrm{mag}$.

The present-day knowledge of the age-metallicity relation in the SMC is mainly based on clusters. Only a few determinations based on stars are available. RR Lyrae star abundances are measured by Smith et al. (1992), while Cepheids metallicity are derived by Harris (1981). The interpretation of the existing SMC age-metallicity relation widely varies from author to author. Current data cannot really discriminate among different models. A continuous enrichment from the oldest to the youngest objects is found by Da Costa \& Hatzidimitriou (1998) and by Dolphin et al. (2001). Olszewski et al. (1996) suggest that no significant enrichment is produced from $10 \mathrm{Gyr}$ ago to 1-2 Gyr ago. At that time the metallicity rapidly increases. Olszewski et al. (1996), Pagel \& Tautvaišienè (1999) and Piatti et al. (2001) analyzes of the data favor a bursting mode of star formation (Harris \& Zaritsky 2004).

In the following, discussing the star formation from field stars, we assume the enrichment history by Pagel \& Tautvaišienė (1999).

\section{Cluster age distribution}

In this section we derive the age distribution of the SMC clusters located in the main body of the SMC using isochrone fitting. The catalog of the studied objects along with coordinates and radii is taken from Bica \& Dutra (2000). 82 clusters and associations are identified in the region centered on NGC 269, while 229 clusters and about 164 associations are studied in the OGLE regions. The cluster list, their coordinates, and the derived ages are available from the authors upon request. In this section, we first describe the method, and we compare the age determination with previous studies, then we present the spatial distribution of clusters of different ages.

\subsection{The method}

The age and the reddening of each cluster are derived by means of isochrone fitting on the CMDs in two ways, by visual inspection and by $\chi^{2}$ minimization. Isochrones are taken from the library of Girardi et al. (2002). The main body of the SMC is a highly crowded region. For this reason, field star contamination severely hampers the age determination. Field subtraction is a critical issue in order to derive the cluster ages. When ages are derived by visual inspection, then field stars are statistically subtracted by the CMDs of the clusters. First we consider an equivalent area of field close to the area of the cluster, but outside the cluster radius given by Bica \& Dutra (2000). Then, the CMDs of both cluster and field are divided in boxes of size $\Delta V=0.5$ and $\Delta(V-I)=0.2$. The incompleteness correction is taken into 
account by dividing the field and cluster CMDs in magnitudecolor bins and then adding on each bin having $N_{\text {th }}$ stars, $\Lambda \times N_{\text {th }}$ objects, where $\Lambda$ is the smallest of the $V$ and $I$ completeness factors. Then, in each box of the cluster CMD, for every field star, the closest cluster star is subtracted. Finally isochrones are superimposed on the CMDs in order to fit the location of the main sequence and of the evolved stars. When the ages are derived using a $\chi^{2}$ minimization, first single stellar populations at changing ages are generated using a Monte Carlo method, taking into account the observational errors on the magnitudes. Then the simulations are corrected for incompleteness, subtracting on each bin $\left(1-\Lambda \times N_{\text {th }}\right)$ objects. The observational field population corrected for the ratio of the field and cluster incompleteness factors is derived as described in the previous paragraph and added to the simulated CMDs. Then the CMDs are divided into bins of 0.2 both in mag and in color and the $\chi^{2}$ function of the difference between the observational CMD and the theoretical ones at changing ages is minimized. A mean metallicity of $Z=0.008$ is assumed, in agreement with observational determination for young objects (Pagel \& Tautvaišienè 1999). However, when the isochrone fitting requires it, a different metal content is adopted. Clusters in which the age values derived in both methods are in reasonable agreement are included in Table 2 and a mean value of the age is given. Table 2 gives the catalog of the clusters, their position, ages, and reddening. Due to the limiting magnitude of the photometry, clusters having turnoff magnitude fainter than $V=21.5 \mathrm{mag}$ in our NGC 269 region data, or $V=20$ in OGLE fields cannot reasonably be identified. This sets a limit of 3 or $1 \mathrm{Gyr}$, respectively (assuming $Z=0.008$ ), to the oldest age we can derive. For homogeneity, we restrict ourselves to study clusters younger than 1 Gyr. To minimize the effect on the age determination of the young clusters and associations due to the saturation limit of the OGLE photometry we make use of the bright star catalog and of the catalog of stars of known spectral type by Massey (2002). To test our method and derive the uncertainties on the age determination, we perform Monte Carlo simulations where synthetic clusters at different ages are generated, field contamination is included and ages are re-derived using $\chi^{2}$ minimization. Ages derived from integrated colors suffer from several effects such as discreteness of isochrones, patchy distribution of the interstellar reddening producing artifacts and spurious peaks in the age distribution (de Grijs \& Anders 2006; Lamers et al. 2006). Those effects are less relevant when ages are derived from CMD fitting. Interstellar extinction plays a minor role on the determination of the ages from the main sequence turnoff location. Uncertainties on the interstellar extinction are of the order of $\Delta\left(A_{\mathrm{V}}\right) \sim 0.1$. In the age range under discussion, this results in an uncertainty of about 0.03 on $\log ($ age $)$. The uncertainties on the age determinations are partly a function of the age itself, in the sense that older clusters are more difficultly identified, and partly a function of the cluster density. $\Delta(\log ($ age $))$, the errors on $\log ($ age $)$ are of the order of 0.22 taking into account the uncertainties on the metal content for relatively dense objects younger than about $2 \times 10^{8} \mathrm{yr}$. A poor age resolution is expected for objects younger than $1 \times 10^{7} \mathrm{yr}$ due to the difficulty of distinguishing massive main sequence and evolved stars, in absence of spectroscopic information. Uncertain membership can further complicate the age determination of those clusters/associations. In addition, the youngest age in the Padova isochrones is $\log (\operatorname{age}(\mathrm{yr}))=6.6$. Objects younger than this limit are therefore assigned to this minimum age. Clusters older than $2 \times 10^{8}$ yr have a mean error $\Delta(\log ($ age $)) \sim 0.3$. This uncertainty is mainly due to the fact that Padova isochrones have problems to reproduce both the turnoff and the luminosity of the clump

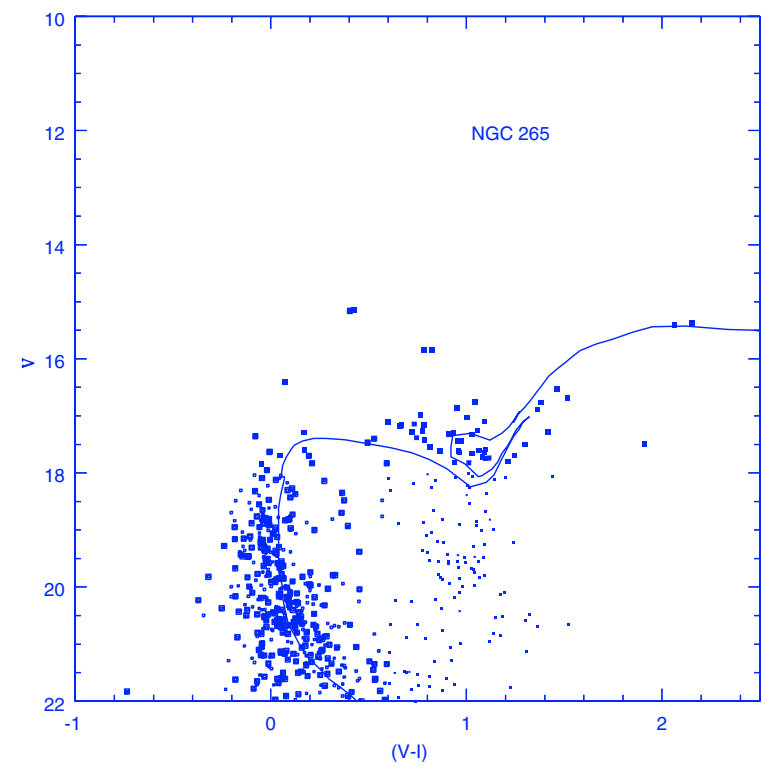

Fig. 4. CM diagram of cluster NGC 265 showing field stars (light dots) and cluster stars (heavy dots). An isochrone having $Z=0.008$ and an age of $2.5 \times 10^{8}$ is over-plotted on the data.

of He-burning stars in the above age range. Very sparse clusters having less than 50 members might have highly uncertain determinations $(\Delta(\log ($ age $)>0.5)$, especially at old ages.

In Fig. 4 we present as example the CMD of NGC 265, one of the bright clusters in the region surrounding NGC 269. The CMD is fitted with an isochrone having $Z=0.008$ and an age of $2.5 \times 10^{8} \mathrm{yr}$. It is evident that it is difficult to reproduce the color of the main sequence and the location of the red evolved stars at once. This is a well known problem. Differential reddening can mimic this effect. However it cannot be excluded that the uncertainties affecting the opacities, and/or the adopted value of the envelope mixing length are responsible of this discrepancy. In Table 2 an index gives the degree of reliability of the age measurement we estimate. Class 1 indicates objects having $\Delta(\log (\operatorname{Age}(\mathrm{yr})))<0.3$; class 2 indicates objects having $0.3<\Delta(\log (\operatorname{Age}(\mathrm{yr})))<0.5$; class 3 indicates objects having $\Delta(\log (\operatorname{Age}(\mathrm{yr})))>0.5$.

In Fig. 5 the cluster ages derived in this paper are compared for the common objects to those obtained via isochrone fitting by Pietrzynski \& Udalski. Our ages are broadly correlated to those presented there. No systematic difference is present. The dispersion about the line 1:1 correlation for the whole sample is $\sigma \log ($ age $)=0.3$. We compare our ages with the compilation by Rafelski \& Zaritsky (2005) where 204 star clusters are identified and their ages are derived using integrated colors. In principle the color of a stellar population provides a reliable chronometer to date clusters. In practice however, as already pointed out by Rafelski \& Zaritsky (2005) stochastic effects on the number of bright stars, uncertainties on the metallicity and on the adopted stellar models make it difficult to precisely infer the cluster formation. Figure 6 shows the comparison between ages derived via isochrone fitting by us and via integrated colors by Rafelski \& Zaritsky (2005) (for $Z=0.004$ ). Neglecting a few outliers, the dispersion around the 1:1 correlation line is $\sigma \log ($ age $) / \log ($ age $)=0.4$. A reasonable agreement is found. The outliers having very large uncertainties are all inside large groups of clusters having small separation along the line of sight or they are very sparse objects. In these clusters to derive star 


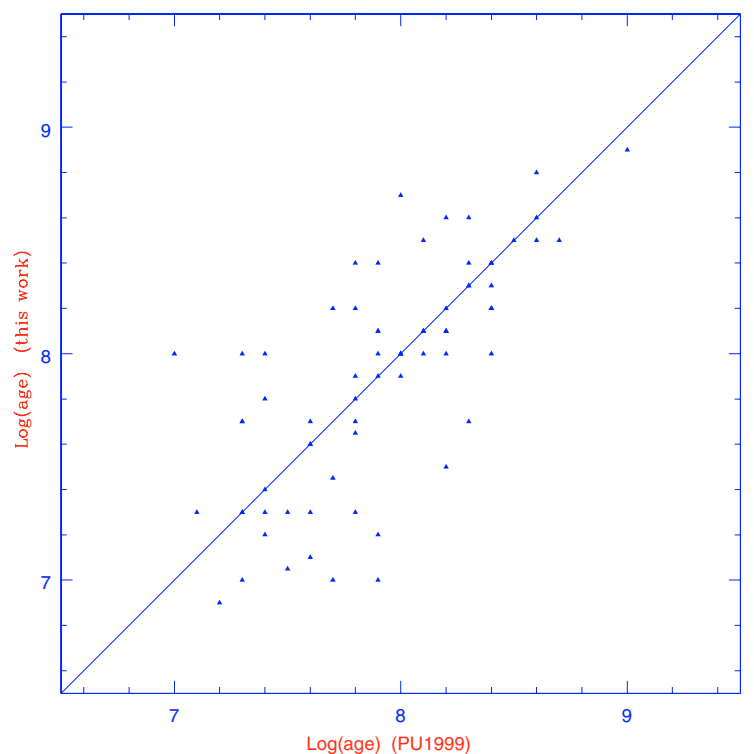

Fig. 5. Cluster ages derived in this paper are compared with the ages by Pietrzynski \& Udalski (PU1999) for the clusters in common. The solid line shows the loci of the 1:1 correlation.

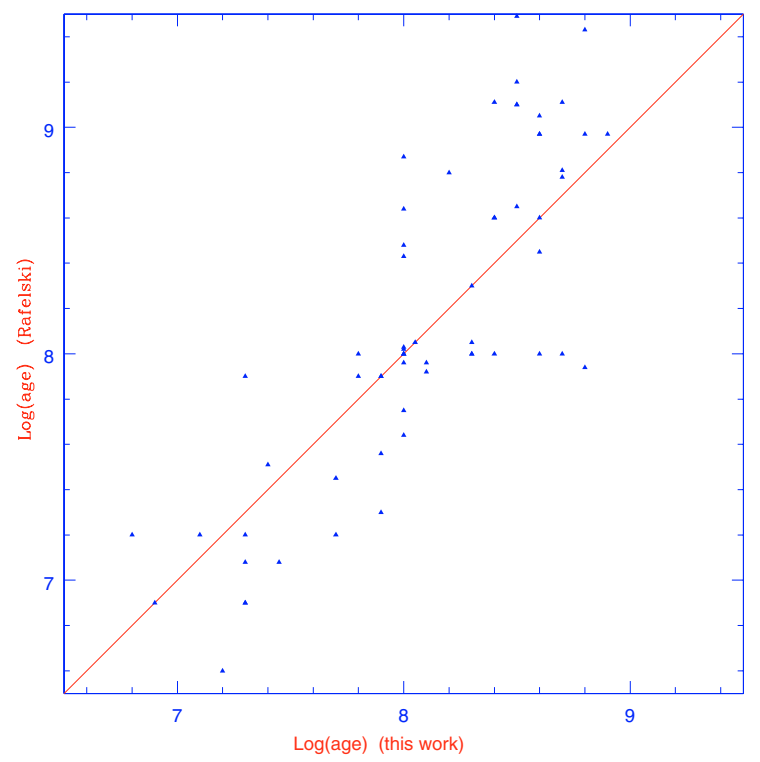

Fig. 6. Cluster ages derived in this paper are compared with the ages by Rafelski \& Zaritsky (2005) (indicated by the label Rafelski) for the clusters in common. The solid line shows the loci of the 1:1 correlation.

memberships on the basis of photometric information is a cumbersome affair.

\subsection{Cluster age distribution in the main body of the SMC}

The age distribution of the clusters in the studied regions is shown in Fig. 7. Two main episodes are found, the first from 5 to $15 \mathrm{Myr}$, the second at $90 \mathrm{Myr}$ about. This latter is followed by an almost constant rate till 1 Gyr. The uncertainties on the age determinations do not allow a finer age resolution. We point out that these peaks are present in the whole sample and in the clusters having the most reliable age measurements (classes 1 and 2 in Table 2). Looking at the spatial distribution of the clusters (see Fig. 8), it is evident that clusters in a given age range are not

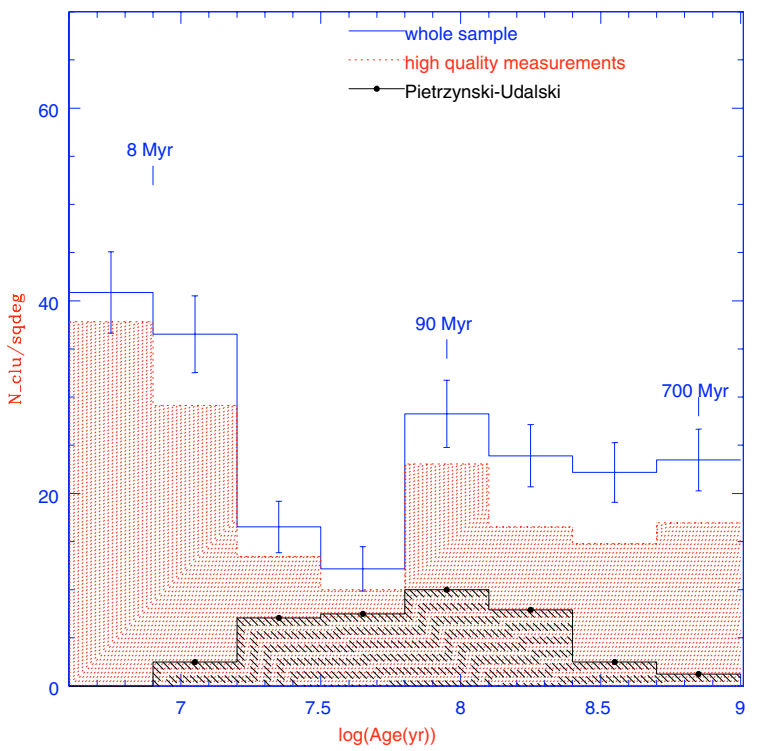

Fig. 7. The cluster age distributions. The continuous line shows the whole sample discussed in this work. The dotted dashed histogram represents the high quality measurements of ages (classes 1 and 2 of Table 2). Squares indicate Pietrzynski \& Udalski (1999) sample.

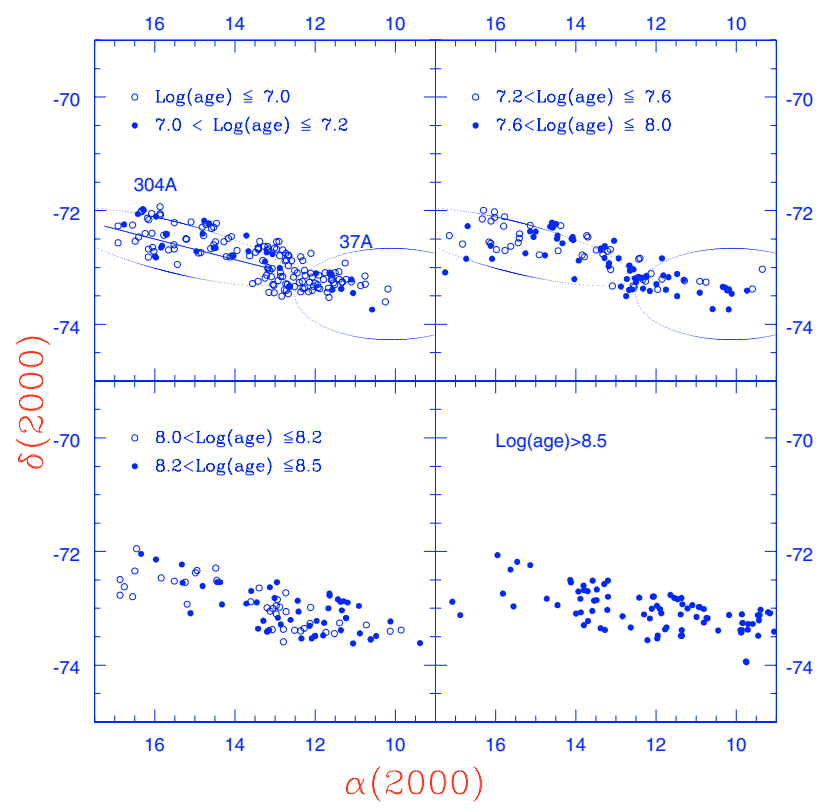

Fig. 8. Spatial distribution of clusters of different ages in the SMC. The approximate locations of the supershells 37A and 304A are shown.

uniformly distributed across the SMC, but the bursts are associated with localized enhanced SFR. Since rotational mixing did not have enough time to smooth the cluster distribution, this points in favor of the fact that these SFR enhancements are not due to artifacts in the age determination procedure, but are likely real.

To describe the spatial distribution of the clusters, we model the SMC disk as an ellipse centered at $\alpha=00^{\mathrm{h}} 52^{\mathrm{m}} 45^{\mathrm{s}}, \delta=$ $-72^{\circ} 49^{\prime} 43^{\prime \prime}$ (J2000) (Crowl et al. 2001) and having an axial ratio $b / a=1 / 2$. Then we define the distance along the major axis as the major axis of the ellipse having the same center and axial ratio and passing on the object. Figure 9 presents the distribution of the clusters per area unit at changing ages as a function of the distance from the SMC center. A complex picture is emerging. 


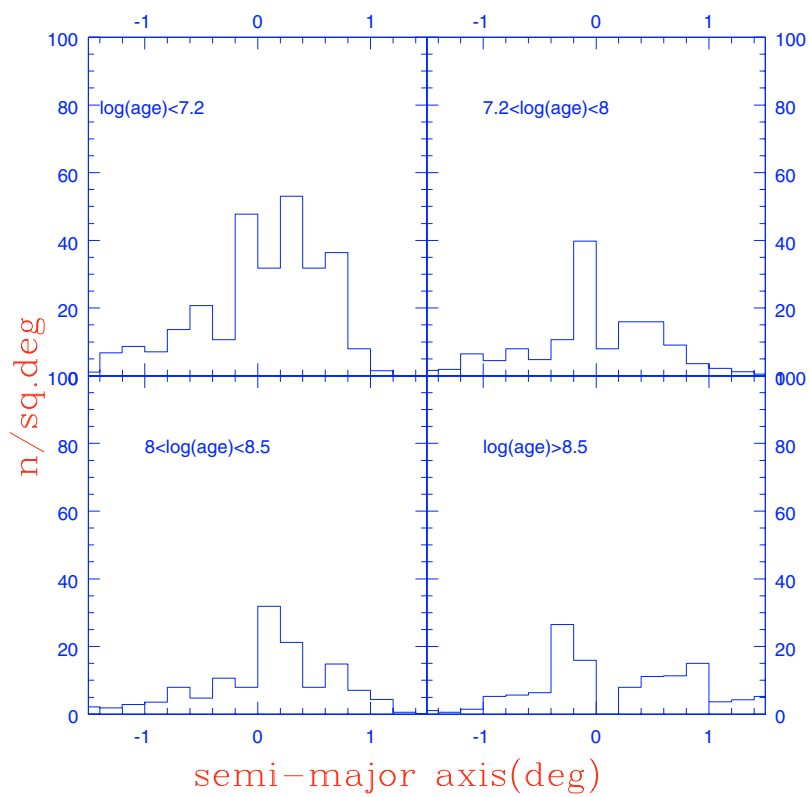

Fig. 9. Cluster distribution as a function of distance from the SMC center at changing ages. Negative axis values indicate the regions East of the center, while positive axis points toward the West.

Two main regions located East and West of the center were active at very young ages $(<15 \mathrm{Myr})$, while the cluster formation process was less significant $100 \mathrm{Myr}$ ago. These regions can be identified with the two HI super-shells 37A and 304A. More detail can be found in the following sections, where the two regions will be analyzed.

Finally, we address the question whether the age distribution we find is representative of the cluster formation rate or whether the tidal field of the SMC was effective in disrupting the less massive clusters. Following the discussion by Boutloukos \& Lamers (2003), in a survey of clusters having a given magnitude limit, two effects contribute to the the age distribution of clusters born at a time $t_{0}$. The first is the fading. Clusters get fainter with time as a result of the evolution of their stars. As a result, the number of observable clusters as a function of age for a given magnitude limit is decreasing. This effect is dominant for young objects. The second is the cluster disruption due to the galactic tidal field and is relevant for old clusters. A steep slope of $\mathrm{d} N_{\text {obs }} / \mathrm{d} t$ is expected. The mass of a cluster decreases almost linearly with time, until the cluster is finally disrupted. This defines the disruption time, $t_{\mathrm{dis}}$. Ignoring burst, the mean cluster formation rate might be assumed as roughly constant. Under this hypothesis, a slope change in the distribution $\mathrm{d} N_{\text {obs }} / \mathrm{d} t$ is expected at a time $t_{\text {cross }}$ where the effects of the disruption begin to be significant. $t_{\text {cross }}$ depends on the photometric evolution of the stellar populations, but is as well a function of the magnitude limit of the cluster sample. Figure 10 presents $\mathrm{d} N_{\text {obs }} / \mathrm{d} t$, the observed age distribution of the SMC clusters. Only objects classified as type C by Bica \& Dutra (2000) are included in the sample. The age distribution of the SMC clusters is rather flat for objects with ages below $\log (t)=8.0$ and decreases steeply at higher ages. This identifies $t_{\text {cross }}$. It cannot be excluded that the sample of clusters we discuss is biased toward dense and massive objects which are more easily recognized than less dense or lower mass objects. However the fact we find a flat distribution at young ages supports the idea that selection effects, fading and incompleteness play a minor role for clusters younger than this limit. In principle $t_{\text {dis }}$ can be derived from $t_{\text {cross }}$ and the slope of the

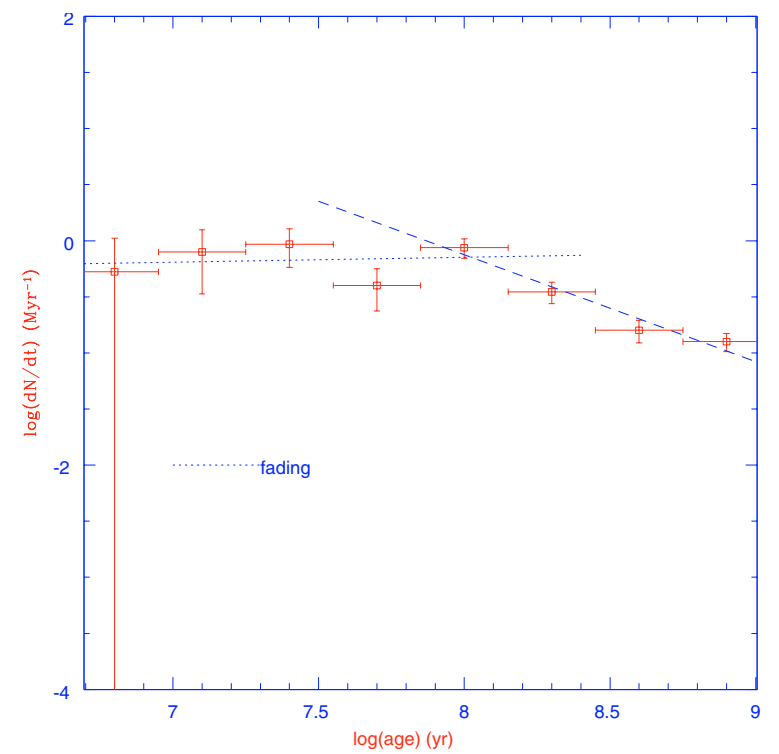

Fig. 10. Cluster age distribution(squares). Only objects classified as $\mathrm{C}$ are included in the sample. The data are fitted by two lines. The dotted line represents the fading line, while the dashed line is the fit for ages where most likely the disruption and the sample incompleteness dominate fading.

disruption line. However in our case, the fact that the completeness correction of the cluster sample is substantially unknown prevents any determination. In fact the sample of ages we derive is probably not complete especially at old ages. We remind that the SMC cluster disruption time derived in literature is of the order of $8 \times 10^{9} \mathrm{yr}$ (de La Fuente Marcos 1997; Boutloukos \& Lamers 2003).

\section{Triggering mechanism of cluster formation}

In this section, we would like to address the complex relation between the clusters and their environment, in order to cast light on the cluster formation process. Several mechanisms of cluster formation have been proposed in literature. Here we quote a few. SMC is believed to have been involved in several perigalactic encounters with the Milky Way and with the LMC, over the past 12 Gyr. The epoch of the most recent perigalactic encounter with the LMC is found to range from $500 \mathrm{Myr}$ ago (Lin et al. 1995) to 200 (Gardiner et al. 1994; Yoshizawa \& Noguchi 2003). At that time the star formation is expected to be enhanced not only in the tidal arms, but also in the main body of the SMC. Clusters are expected to form as a result of relatively high velocity cloud-cloud collisions (Zhang et al. 2001; Bekki et al. 2004). This mechanism is particularly efficient during galaxy interactions and mergers. The value of the turbulent velocity between clouds that can give rise to cluster formation is a highly uncertain parameter, going from $50-100 \mathrm{~km} \mathrm{~s}^{-1}$ (Zhang et al. 2001) to $20-30 \mathrm{~km} \mathrm{~s}^{-1}$ (Bekki et al. 2004). Alternatively, high speed motions may produce a high-pressure environment that in turn, can trigger turbulence or shocks (Elmegreen \& Efremov 1997). Finally, star formation might be triggered by stellar winds and supernovae explosions through compression by turbulent motions (Larson 1993).

The correlation, if any, between young star clusters and their environment can cast light on the cluster formation process. In the following, we first discuss the age distribution in the regions of the two super-shells. Then we discuss the degree of clustering of the clusters and young field stars. Finally, we compare the 
spatial distribution with the column density of the gas observed in the $\mathrm{HI}$ and with the dispersion velocity field.

\subsection{Super-shells and cluster formation}

The interstellar medium of the SMC shows a fractal structure, consisting of a hierarchy of HI clouds and shells. Kinematic studies of the HI data have revealed the presence of two supershells in the SMC disk, namely 37A and 304A (Stanimirovic et al. 1999) which may consist of smaller superimposed shells. The true origin of the holes and small shells in the interstellar medium is still under discussion. There is evidence that at least $10 \%$ of the small shells found in the SMC are not associated with young star formation. For this reason, it is unlikely that those few shells are the result of supernova explosions (Hatzidimitriou et al. 2005) but they might be due to turbulence and gravitational instabilities (Dib \& Burkert 2005). However the vast majority of the shells and supershells is associated with young objects and it is probably formed in the standard way, because of the combined effects of supernovae and stellar winds (McCray \& Kafatos 1987). Inside the shells, sequential or secondary star formation is expected to be triggered by supernova explosions. In the following we will discuss the spatial distribution of the clusters/associations of different ages inside 37A and 304A to clarify the relation between cluster formation and super-shells.

\subsubsection{Cluster age distribution in the region of the supershell 37A}

In order to bring into evidence the spatial distribution of the clusters of different ages, we plot the age distribution of the clusters against the distance from the center. As we did in the previous section, we approximate the shell with an ellipse whose center and axial ratio are given by Stanimirovic et al. (1999). For sake of clarity we remind that the supershell is centered at $\alpha=00^{\mathrm{h}} 40^{\mathrm{m}} 26^{\mathrm{s}}$ and $\delta=-73^{\circ} 28^{\prime} 06^{\prime \prime}$ and has a major axis $a=840 \mathrm{pc}$ (or $0.8 \mathrm{deg}$ ), axial ratio of 0.89 and a position angle of $160^{\circ}$ (Stanimirovic et al. 1999). Because of their position angle, the minor axes of the ellipses are roughly aligned in the direction E-W. Figure 11 presents the cluster age distribution as a function of the semi-minor axis. Our data do not completely cover the supershell at the western side, but the vast majority of it is included in the data. While objects older than about 15 Myr are found East and West of the center, a discontinuity in the spatial distribution of younger clusters is present. They are almost all located toward the eastern rim of the supershell 37A where gas and dust are located (Staveley-Smith et al. 1997; Stanimirovic et al. 1999). Only two clusters as old as $15 \mathrm{Myr}$ are found West of the center. This discontinuity clearly indicates the epoch of the shell formation which was preceded by a period of relative quiescence. This determination of the supershell age is in agreement with the dynamical age of $17 \mathrm{Myr}$ (Stanimirovic et al. 1999).

It is quite difficult to ascertain the presence of secondary or propagating star formation events as it is expected in the standard model by McCray \& Kafatos (1987) if stellar winds and supernovae explosions were responsible for the formation of the supershell. Standard model of shell formation predicts that young objects are located at the edges, while older stars are more centrally concentrated. The fact that objects younger than the dynamical estimate of the supershell age are located toward the eastern rim of the supershell, while older clusters are widely distributed might be interpreted as a mild age gradient. However,

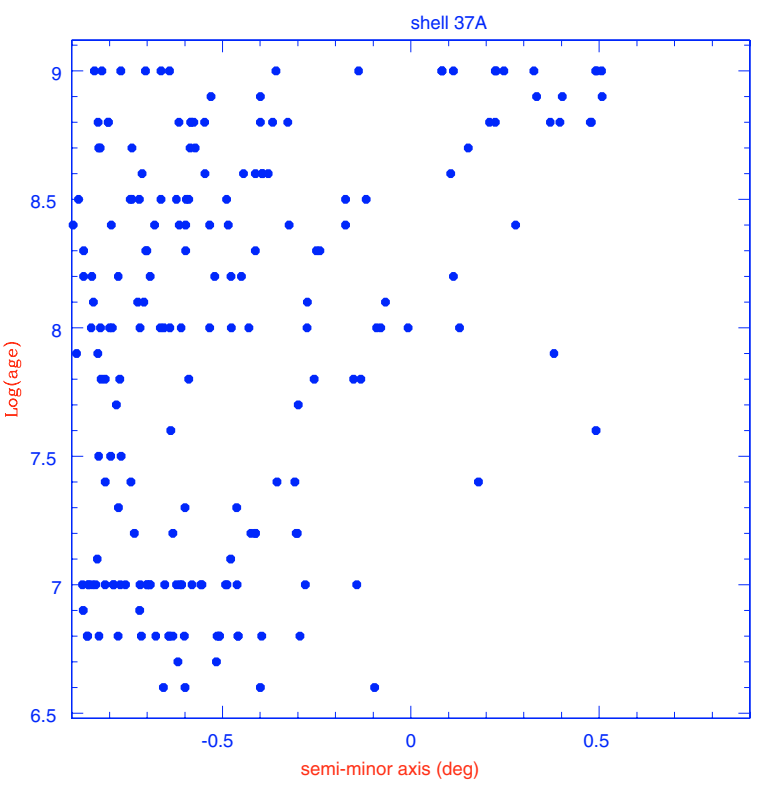

Fig. 11. Age distribution inside the supershell 37A plotted against the semi-minor axis (see text for details). Negative axis refers to the eastern part of the shell.

we remind that the whole analysis is complicated by the extension of the SMC along the line of sight. The apparent distance of the clusters from the center might be due to projection effects.

A quantitative description of the cluster age distribution is shown in Fig. 12. We subdivide the supershell region in two parts, one West, from $\alpha=00^{\mathrm{h}} 44^{\mathrm{m}} 00^{\mathrm{s}}$ (or 11 degrees) to $\alpha=$ $00^{\mathrm{h}} 36^{\mathrm{m}} 00^{\mathrm{s}}$ (or 9 deg.) and one East of $\alpha=00^{\mathrm{h}} 44^{\mathrm{m}} 00^{\mathrm{s}}$. The age distribution presents two episodes that might have different origin. Looking at the cluster age distribution, (see Fig. 12) it is evident that the epoch of the shell formation is coincident with an enhancement in the cluster formation rate in the eastern part.

In addition to this young episode, the cluster age distribution at the eastern part shows an enhancements between 80 and $400 \mathrm{Myr}$. On the western side the cluster formation was less efficient at ages younger than $15 \mathrm{Myr}$, while it was comparable at older ages.

\subsubsection{Cluster age distribution in the region of the supershell 304A}

Analogously to what we did in previous section, we approximate the supershell region with an ellipse and discuss the distribution of the clusters inside homologous ellipses having the same center and axial ratio of the shell. We remind that the supershell is centered at $\alpha=01^{\mathrm{h}} 02^{\mathrm{m}} 16^{\mathrm{s}}$ and $\delta=-72^{\circ} 38^{\prime} 12^{\prime \prime}$ and has a major axis $a=910 \mathrm{pc}$ (or $0.87^{\circ}$ ), axial ratio of 0.45 and a position angle of $80^{\circ}$ (Stanimirovic et al. 1999). Figure 13 shows the cluster distribution as a function of the distance from the center calculated along the major axis of the ellipse representing the supershell (see previous section). The distribution of the clusters along the major axis (approximately oriented in the direction $\mathrm{W}$ E) clearly shows that at any ages the star formation took place preferentially in the western part of the shell, in the direction of the shell $37 \mathrm{~A}$. An almost symmetric distribution is visible for ages younger than $20 \mathrm{Myr}$, while older objects are mainly located West of the center. This epoch roughly indicates the time of the formation of the shell and is in agreement with the dynamical age of the supershell of 14 Myr (Stanimirovic et al. 1999). 


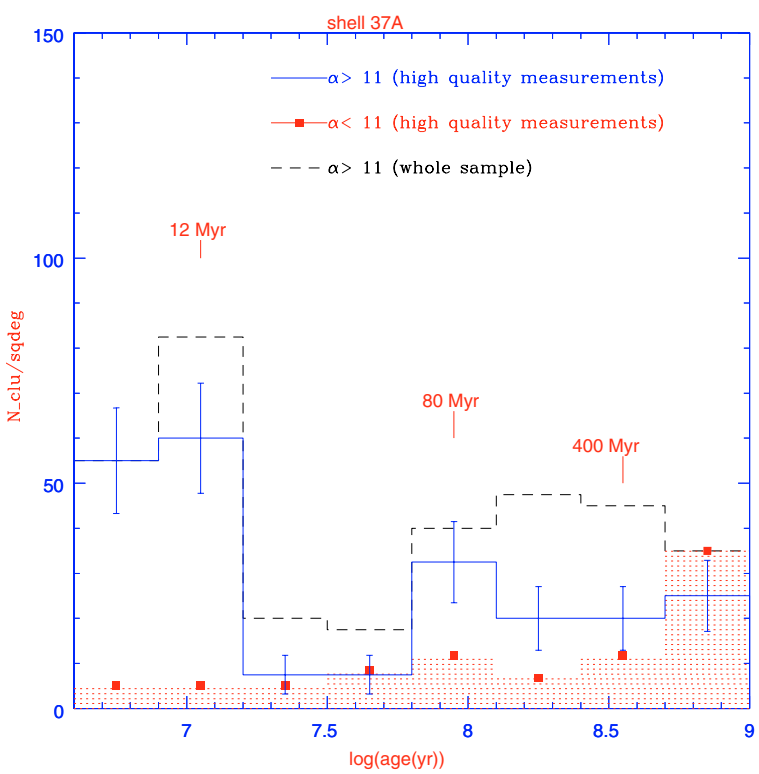

Fig. 12. Dashed line is the age distribution of the whole cluster sample on the eastern side of the shell 37A $\left(\alpha>11 \mathrm{deg}\right.$ or $\left.\alpha<00^{\mathrm{h}} 44^{\mathrm{m}} 00^{\mathrm{s}}\right)$. Continuous line is the analogous for high quality measurements (see text for details). Squares show the cluster age distribution on the western side of the shell 37A ( $\alpha<11 \mathrm{deg})$ for clusters having high quality measurements of the age. Error bars indicate the Poissonian uncertainties on the counts.

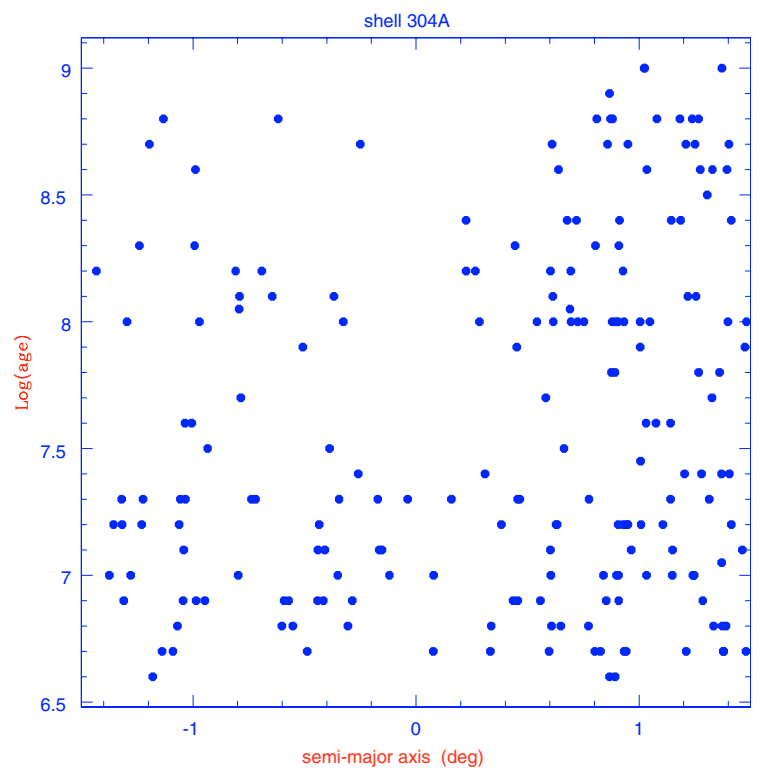

Fig. 13. Age distribution inside the supershell 304A plotted against the semi-major axis (see text for details). Negative values of the semimajor-axis indicate objects located East of the center, while positive values refer to clusters West of the center.

A quantitative description of the age distribution is derived subdividing the region in two parts defined by the line drawn in Fig. 8, roughly separating the northern region from the Southern. Figure 14 presents the age distribution of the clusters. The star formation was more active in the northern region, where the majority of the $\mathrm{H}_{\alpha}$ emission is located. The cluster distribution in the supershell 304A region shows a continuous formation from a few Myr to 1 Gyr with enhancements from a few Myr to $15 \mathrm{Myr}$, and at 90 . The youngest episode is in coincidence with the epoch of the formation of the supershell.

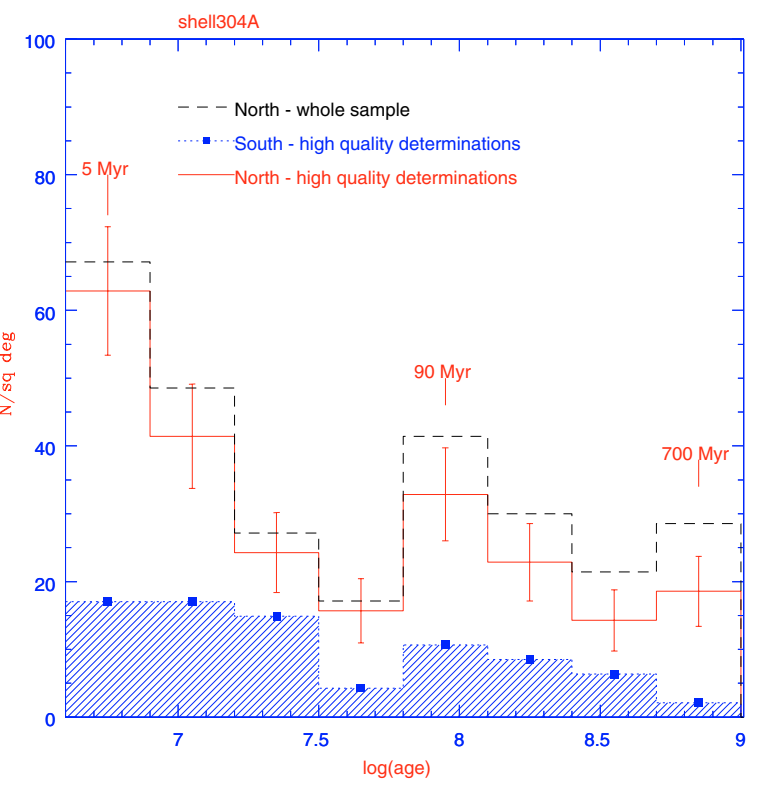

Fig. 14. Dashed line shows the cluster age distribution for the whole sample of objects inside the shell 304A North of the line drawn in Fig. 8 where the large regions of $\mathrm{H}_{\alpha}$ emission are located. Continuous line is the analogous for clusters having high quality determinations of the age, while dashed histogram is the analogous at the Southern side. Error bars indicate the Poissonian uncertainties on the counts.

Summarizing this section, the two super-shells are clearly visible in the age distribution of the clusters. From the epochs of their formation up to now, an enhancement in the cluster formation took place. It is evident that the same mechanism (SN explosions, stellar winds, turbulence) producing the shells in the gas distribution is responsible of the formation of the objects younger than 15-20 Myr. The super-shells probably formed in more complex way than what is described by the simple model by McCray \& Kafatos (1987): even if young objects are more numerous at the edges of the shells, a clear age gradient from the centers to the rims is not evident. The inter-shell region was specially active, possibly due to compression phenomena related to the expansion of the shells. Finally, an episode at about $90 \mathrm{Myr}$ is found in both shells, even if it is more relevant in the shell 304A. This latter is possibly related to the most recent epoch of close interaction between SMC and LMC.

\section{Correlation of the clusters with their environment}

In this section we first make use of the correlation function to discuss the degree of clustering of the objects and derive the spatial scale of the formation. Then we explore the correlation between the clusters and their environment, in particular the HI flux and velocity dispersion, and the location of the $\mathrm{CO}$ clouds.

We adopt the two-point correlation function as a description of the distribution of clusters and associations with the HI flux map by Stanimirovic et al. (1999). This allows a quantitative measurement of the clustering to complement the visual exploration of the maps (see following). $\xi(r)$, the autocorrelation function, is defined using the probability $1+\xi(r)$ of finding a neighbor in a shell element of volume $\mathrm{d}^{3} r$ at a distance $r$ from an object of the sample as:

$1+\xi(r)=1 /(N n) \sum_{i=1}^{N} n_{i}(r)$ 


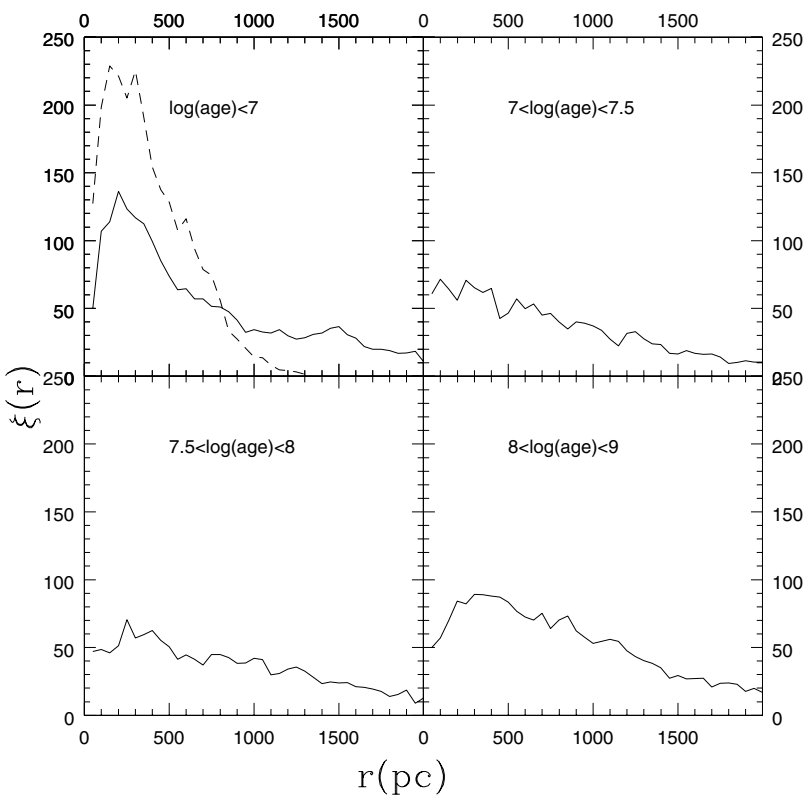

Fig. 15. Autocorrelation function of clusters (solid line) and field stars younger than $10 \mathrm{Myr}$ (dotted line).

where $n_{i}(r)$ is the number density of objects found in an annulus centered on the $i$ th object and having radius between $r$ and $r+\mathrm{d} r, N$ is the total number of objects, and finally, $n$ is the average number (Peebles 1980). A Monte Carlo algorithm is used to derive the area included in the data when the annulus extends outside the studied region.

When the clusters are associated with a continuous map, then the cross correlation function is defined as:

$1+\xi(r)=1 /(N f) \sum_{i=1}^{N} f_{i}(r)$

where $f_{i}(r)$ is the average flux in an annulus with radius $r$ centered on the object, and $f$ is the average flux over the whole region. Using the above definitions, a random distribution of clusters will produce a flat correlation, with $\xi(r) \sim 0$. A peaked $\xi(r)$ at small radii indicates a positive correlation, and the full width half maximum of the peak itself represents the spatial scale of the association between the cluster distribution and the flux. The absolute value of $\xi(r)$ is a measure of the concentration of the flux at a given distance $r$ from the cluster center, relative to the average flux. Since only clusters in the disk of the SMC are under discussion, the regions of the HI map outside the OGLE fields are masked out.

\subsection{Clustering of star clusters and field stars}

Figure 15 presents the autocorrelation function of the clusters. Objects younger than $10 \mathrm{Myr}$ show a peaked distribution with half maximum full width of the order of $500 \mathrm{pc}$. This distribution might reflect the structure of the interstellar medium from which they formed. The scale of the clustering is larger than the typical size of molecular clouds in the SMC and LMC which is going from about 10 to $100 \mathrm{pc}$ (Israel et al. 2003) but is comparable to the size of the complexes and groups of molecular clouds found in the LMC and SMC (Mizuno et al. 2001; Israel et al. 2003). The autocorrelation function of the clusters is getting flatter with age, implying a weaker correlation. In fact, because of cinematic effects, older clusters are more spread out than young objects. In order to verify whether clusters and field stars show evidence of different formation mode, we compare the distribution of the young clusters and young field stars in the SMC disk using the bright star survey by Massey (2002). We select only field stars younger than 10 Myr having $V<14$. Figure 15 presents the autocorrelation function of the bright field stars showing the same spatial scale as the clusters, but a higher degree of correlation.

\subsection{Cross-correlation of the cluster distribution with HI map}

The HI 21-cm emission line provides a measurement of the content and kinematics of the atomic hydrogen. Figure 16 compares the HI map by Stanimirovic et al. (1999) with the location of the young clusters. The region of the maximum HI intensity is located at $\alpha=00^{\mathrm{h}} 47^{\mathrm{m}} 33^{\mathrm{s}}$ and $\delta=-73^{\circ} 05^{\prime} 26^{\prime \prime}$ and shows cluster formation at the edges, where the rim of the supershell $37 \mathrm{~A}$ is located. A quantitative measurement of the degree of correlation between clusters and HI is presented in Fig. 17 showing the correlation function at different ages. Clusters younger than $10 \mathrm{Myr}$ show a positive correlation with the HI map. The degree of correlation is decreasing with age. The correlation is the weakest for clusters in the age range 300-1000 Myr, with a plateau in $\xi(r)$ in the inner $250 \mathrm{pc}$, implying that the clusters are located away from the peaks of the HI. This positive correlation confirms that the formation of clusters is related to the presence of atomic gas.

\subsection{Comparing cluster distribution and $\mathrm{HI}$ velocity dispersion}

A comparison between the cluster distribution and the velocity field provides additional information about the formation and evolution of young clusters. Tidal interaction between the SMC and LMC/MW involves a motion of the gas, which in turn might cause shocks and trigger star and cluster formation. The quasi periodic approaches of the SMC to the LMC sustain tidally and hydrodynamically the large scale turbulent motions in the gas (Kumai et al. 1993). In fact the SMC gas is found to present large scale motion elongated on the line of sight (Mathewson et al. 1986; Stanimirovic et al. 1999). On the other hand, supernovae explosion and stellar winds from young clusters can put energy into the interstellar medium influencing the motion of the gas. So a correlation between clusters and high velocity dispersion of HI can cast light on both the cause and the effects of the cluster formation. We compare in Fig. 18 the velocity dispersion field of the HI in SMC (Stanimirović et al. 2004) with the location of the clusters of different ages. If clusters are formed in high velocity gas motion, then we expect a correlation between the position of young objects and the velocity dispersion of $\mathrm{HI}$.

The super-shells 37A and 304A are clearly visible as disturbances on the velocity field. In the shell 304A about $48 \%$ of the clusters younger than $10 \mathrm{Myr}$ are apparently located in the high dispersion regions $\left(\sigma_{v}>25 \mathrm{~km} \mathrm{~s}^{-1}\right)$. The percentage of objects related to high velocity motion is still $45 \%$ in the age range $20-100 \mathrm{Myr}$, and is about $35 \%$ at ages of $1 \mathrm{Gyr}$. In the shell 37A clusters and associations younger than $10 \mathrm{Myr}$ are found in regions of intermediate velocity $\left(\sigma_{v} \sim 15-25 \mathrm{~km} \mathrm{~s}^{-1}\right)$. Only the $8 \%$ of young clusters is apparently located in the high velocity regions. Surprisingly, about $30 \%$ of clusters in the age range 100-1000 Myr seems to be correlated with high velocity motions, which might be due to chance superposition along the line of sight. If we assume that the distribution of the clusters is uniform, then the probability of a chance superposition is given by the ratio of the area of the high velocity regions over the 

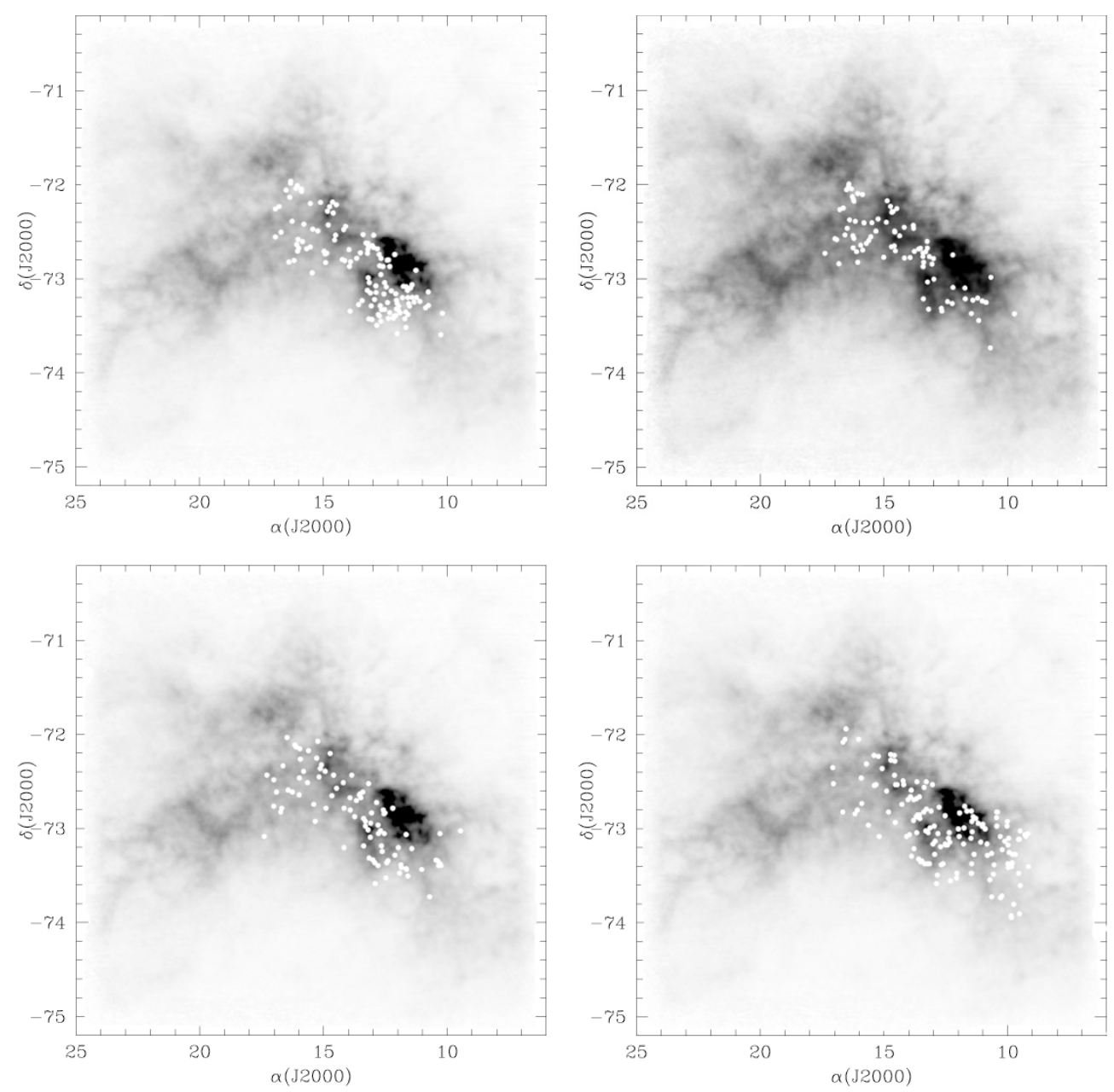

Fig. 16. The HI column intensity map in SMC is compared with the location of clusters of different ages. Top left panel refers to object younger than $10 \mathrm{Myr}$; top right panel presents clusters in the age range 10-30 Myr; bottom left panel shows the objects having ages going from 30 to $100 \mathrm{Myr}$; bottom right presents clusters from 100 to 1000 Myr old.

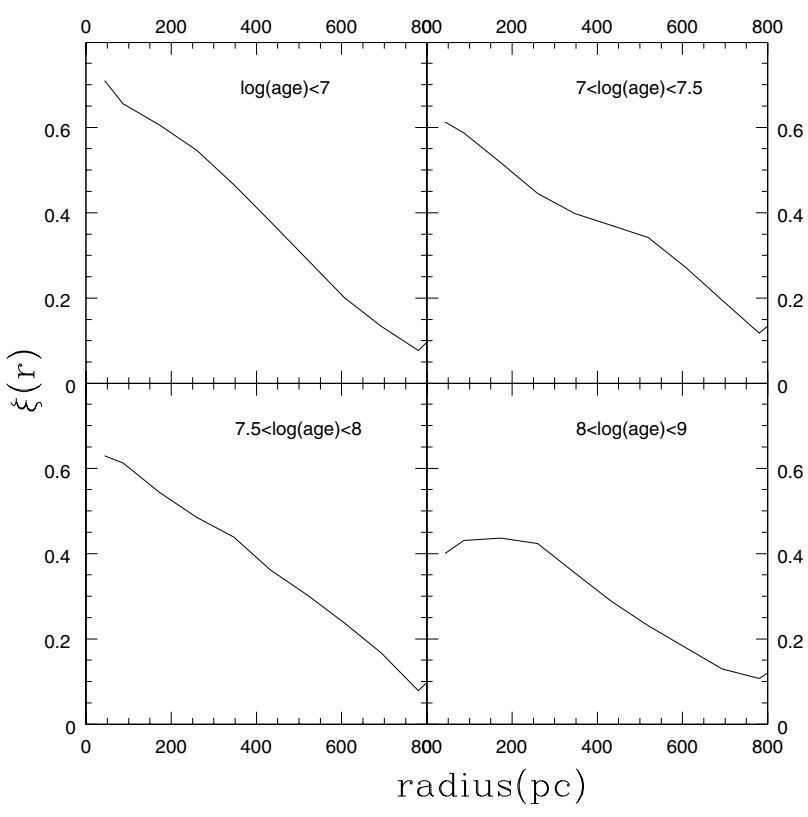

Fig. 17. Correlation function between clusters and HI flux map.

total disk area. Under this assumption, the probability of a chance superposition is of the order of $32 \%$.
Summarizing this section, cloud collisions might have triggered cluster formation in the region of 304A. However this mechanism seems not to account for the formation of clusters in $37 \mathrm{~A}$.

\subsection{CO clouds}

Finally, to derive more information about the recent SF process, we compare the distribution of the clusters and of the $\mathrm{CO}$ clouds. $\mathrm{CO}$ emission provides a probe of molecular gas inside the galaxies, since it is related to $\mathrm{H}_{2}$ column density. Rubio et al. (1993), Mizuno et al. (2001) reveal the presence of two main complexes of CO molecular clouds located in the South West $\alpha=0^{\mathrm{h}} 45^{\mathrm{m}}$ and $\delta=-73^{\circ} 35^{\prime}$ and North East at $\alpha=1^{\mathrm{h}} 00^{\mathrm{m}}$ and $\delta=-72^{\circ} 20^{\prime}$. They find that the location of Giant Molecular Clouds (GMCs) shows a good spatial correlation with the HII regions and young emission clusters indicating that cluster formation is ongoing.

We compare the age distribution with the $\mathrm{CO}$ cloud catalog by Mizuno et al. (2001) in the SW region of the disk. This region is located at the eastern rim of the supershell 37A. We find that about $20 \%$ of the clusters older than $10 \mathrm{Myr}$ are located close to the $\mathrm{CO}$ emission (Fig. 19). The percentage of associated clusters becomes $35 \%$ when younger objects are considered. Assuming a uniform cluster distribution, the probability of a chance superposition is of the order of $11 \%$ (Mizuno et al. 2001). A significant fraction of the objects younger than $10 \mathrm{Myr}$ are probably 

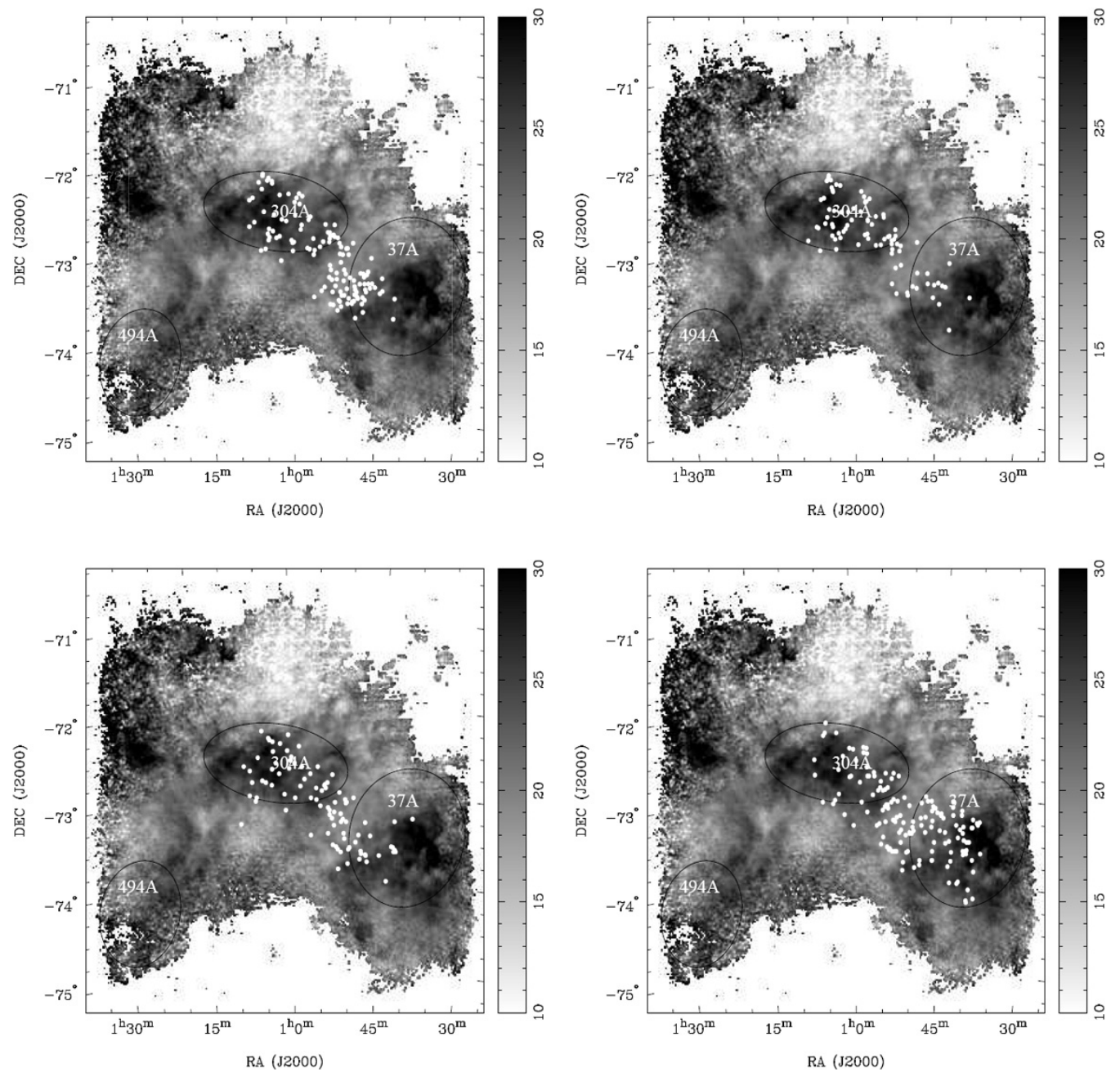

Fig. 18. The velocity dispersion field of the HI in SMC taken by Stanimirovic et al. (2004) is compared with the location of clusters of different ages. Top left panel refers to object younger than $10 \mathrm{Myr}$; top right panel presents clusters in the age range 10-30 Myr; bottom left panel shows the objects having ages going from 30 to $100 \mathrm{Myr}$; bottom right panel presents clusters from 100 to 1000 Myr old.

physically associated with the clouds. Our result implies a rapid dissipation of the $\mathrm{CO}$ clouds. This is in agreement with the evolutionary time scale of the giant molecular clouds derived in the LMC by Fukui (2005). Figure 20 compares the location of the clouds with field stars younger than $10 \mathrm{Myr}$ (or brighter than $V=14$ ) taken from the catalog by Massey (2002). The $38 \%$ of the stars younger than $6 \mathrm{Myr}$ (or brighter than $V=13$ ) are associated with the clouds, while only $25 \%$ of the stars in the age range 6-10 Myr are located in the proximity of the $\mathrm{CO}$ emission. This gives further support to the idea that the formation of young clusters and field stars is closely related to the $\mathrm{CO}$ clouds. In addition, about $70 \%$ of the stars younger than $10 \mathrm{Myr}$ and associated with the clouds are located close to the eastern side of the clouds themselves. We point out that the SW CO clouds are located toward the interface between the two super-shells, 37A and 304A: dynamical effects due to the expansion of the supergiant shells may be important in triggering the compression of the molecular clouds and the formation of new stars. Figure 20 might indicate that young stars are more easily found at the compression edge of the clouds. This effect is more relevant for field stars, while is not obvious for the cluster distribution. The northern region studied by Mizuno et al. (2001) where CO clouds are found is not included in OGLE data. Figure 21 shows the location of field stars brighter than $V=14$ and of the CO clouds in this region. The data are from Massey (2002). Only a negligible percentage of stars is apparently associated to the $\mathrm{CO}$ clouds. This is in agreement with Mizuno et al. (2001) who find a substantial difference between the CO cloud complexes in the SW and in the North of the disk. In the northern region, the clouds are smaller in size than those of SW region. No particular correlation is found with the distribution of young associations and clusters except for the objects associated with N66. In our Galaxy massive stars are formed in clusters in giant molecular cloud (GMC) complexes and almost no GMCs are found in the solar vicinity lacking of massive young stars (Fukui 2005). Discussing the distribution of CO clouds in the LMC, Fukui et al. (1999) and Fukui (2005) find a different behavior, classifying the giant molecular clouds in three groups: 1) without massive OB stars (which does not exclude that low mass stars are formed); 2) associated with small HII regions; 3) associated with clusters and large HII regions. About $38 \%$ of the molecular clouds in the LMC are not related to recent massive star formation. If $\mathrm{CO}$ emission surveys over larger areas confirm this finding, then the properties of massive star formation regions are probably different in the Milky Way from those in the MCs.

\section{Field star formation}

In this section we derive the star formation rate of the field stars in order to compare it with the cluster formation epochs. 


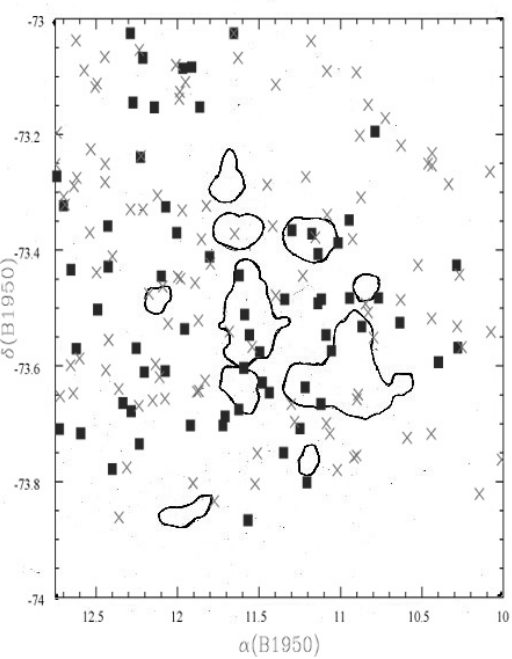

Fig. 19. The distribution of clusters and associations younger than $10 \mathrm{Myr}$ (squares), and clusters older than $10 \mathrm{Myr}$ (crosses) is compared with the approximate location and size of the CO clouds (heavy solid line) from Mizuno et al. (2001) in the region of the shell 37A.

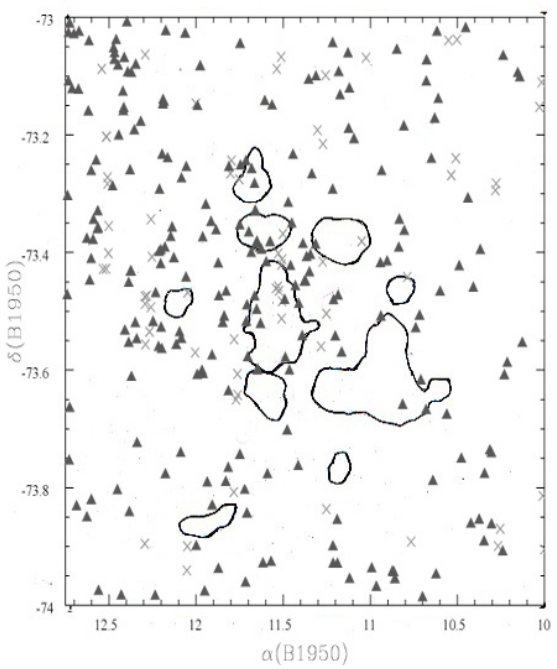

Fig. 20. The distribution of field stars younger than $6 \mathrm{Myr}$ (crosses), and stars in the age range 6-10 Myr (triangles) is compared with the approximate location and size of the $\mathrm{CO}$ clouds (heavy solid line) from Mizuno et al. (2001) in the region of the shell 37A.

The goal is to verify whether field objects and clusters follow similar modes of formation. We make use of our data inside the supershell 37A and of OGLE data in the rest of the disk. In the following, we first present the method, then we discuss the star formation history of the field population in the two super-shells, comparing the periods of enhancements with what we derive for the clusters.

\subsection{The method}

To infer the SFR of this galaxy, theoretical CMDs in different age ranges are simulated. Simulations include the spread due to observational photometric errors and reddening as described in the previous sections. For each age bin, from 10000 to 15000 stars were generated down to the photometric completeness limit. The generation of the synthetic populations makes use of the set of stellar tracks by Girardi et al. (1996, 2000). The constancy of

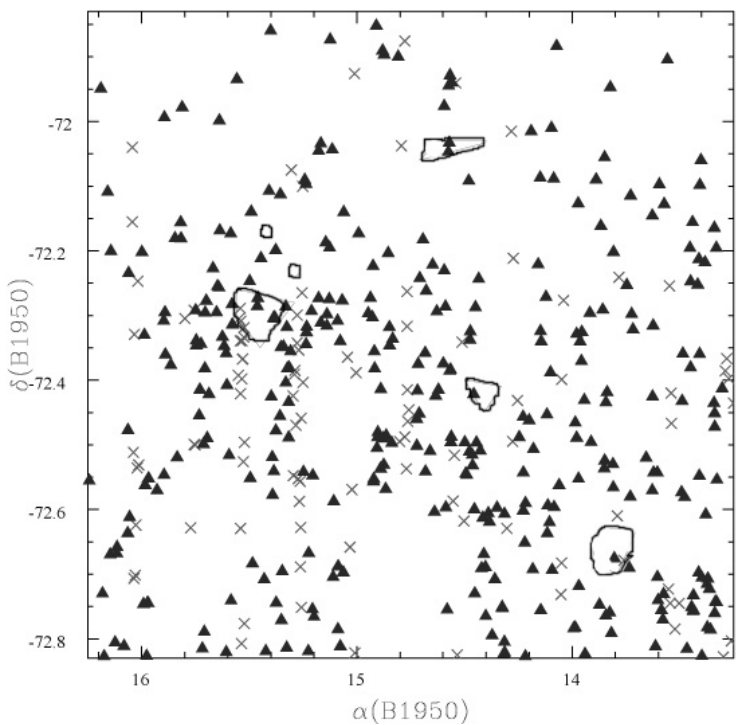

Fig. 21. The distribution of field stars younger than 6 Myr (crosses), and stars in the age range 6-10 Myr (triangles) is compared with the approximate location and size of the $\mathrm{CO}$ clouds (heavy solid line) from Mizuno et al. (2001) in the northern region of the disk.

the initial mass function (IMF) slope in different environments is still a matter of discussion, although a number of recent papers proposed the idea of a universal IMF (Kroupa \& Boily 2002; Wyse et al. 2002; Chabrier 2003; Weidner \& Kroupa 2004). The determination of the IMF is beyond the scope of this paper. Here the IMF of Kroupa $(2001,2002)$ is assumed. This IMF is a power-law function with a slope $x=2.3$ for stellar masses $m>0.5 M_{\odot}$, while $x=1.3$ in the mass range $0.08-0.5 M_{\odot}$ (when the standard Salpeter value is 2.35).

The completeness of the data is taken into account by dividing the simulated CMD in magnitude-color bins and then subtracting from each bin having $N_{\text {th }}$ stars, $(1-\Lambda) N_{\text {th }}$, where $\Lambda$ is the smallest of the $V$ and $I$ completeness factors as given in Fig. 2.

Finally, the SFR is derived by means of a downhill simplex method (Harris \& Zaritsky 2004), minimizing the $\chi^{2}$ function in a parameter-space having $N$ dimensions. At each step the local $\chi^{2}$ gradient is derived and a step in the direction of the gradient is taken, till a minimum is found. In the following, the observational CMD is divided into bins. Recent work concerning the determination of the SFR from the CMDs has pointed out the importance of using a binning, that takes into account the various stellar evolutionary phases, as well as the uncertainties on the stellar models (Rizzi et al. 2002). In Sect. 4 we show that problems arise when isochrones are used to fit the main sequence and the He-burning stars. For this reason, while a coarser magnitude bin distribution is used on the main sequence, only a few bins are considered for the red evolved stars. This avoids that the uncertainties on both the observational errors and the theoretical models (i.e. on bolometric corrections, RGB and AGB location, extension of the core He-burning loop) result in spurious solutions. For this reason, we are not very sensitive to the SFR at ages older than 1 Gyr. This is illustrated in Fig. 22. To prevent settling on local rather than global minima, the simplex is first started from a random position, then when a possible solution is obtained, it is re-started from a position very close to it. Finally, when a minimum is found 30000 random directions are searched for a new minimum. 


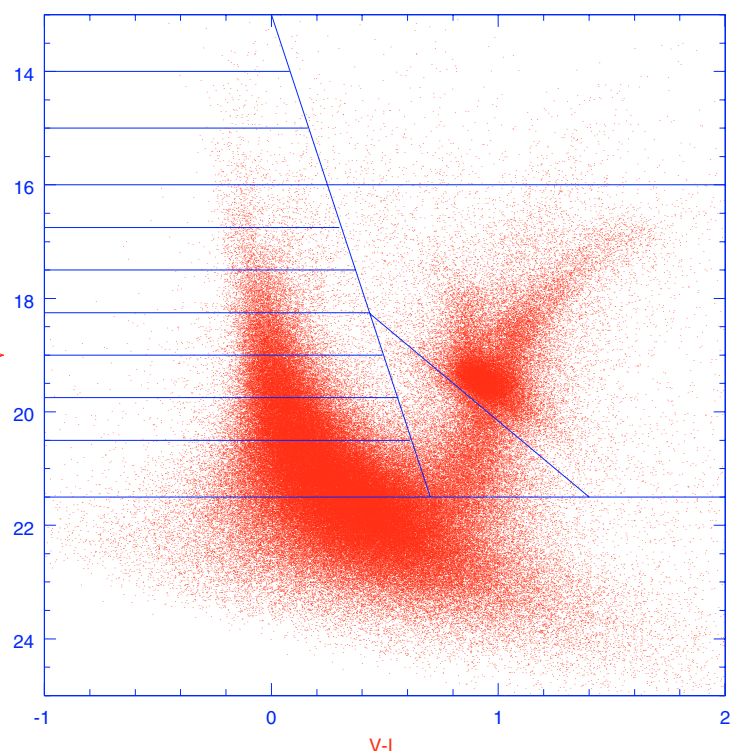

Fig. 22. Bin division of the CMD.

Table 1. Synthetic population age and metallicity.

\begin{tabular}{cc}
\hline \hline Age & $Z$ \\
\hline $5 \mathrm{e} 6: 1.5 \mathrm{e} 7$ & 0.006 \\
$1.5 \mathrm{e} 7: 2.5 \mathrm{e} 7$ & 0.006 \\
$2.5 \mathrm{e} 7: 4.0 \mathrm{e} 7$ & 0.006 \\
$4.0 \mathrm{e} 7: 6.3 \mathrm{e} 7$ & 0.006 \\
$6.3 \mathrm{e} 7: 1.0 \mathrm{e} 8$ & 0.006 \\
$1.0 \mathrm{e} 8: 1.6 \mathrm{e} 8$ & 0.006 \\
$1.6 \mathrm{e} 8: 2.5 \mathrm{e} 8$ & 0.006 \\
$2.5 \mathrm{e} 8: 4.0 \mathrm{e} 8$ & 0.006 \\
$4.0 \mathrm{e} 8: 6.3 \mathrm{e} 8$ & 0.006 \\
$6.3 \mathrm{e} 8: 1.0 \mathrm{e} 8$ & 0.006 \\
$1.0 \mathrm{e} 9: 1.6 \mathrm{e} 9$ & $0.001: 0.006$ \\
$1.6 \mathrm{e} 9: 2.5 \mathrm{e} 9$ & $0.001: 0.003$ \\
$2.5 \mathrm{e} 9: 4.0 \mathrm{e} 9$ & $0.001: 0.003$ \\
$4.0 \mathrm{e} 9: 6.0 \mathrm{e} 9$ & $0.001: 0.003$ \\
$6.0 \mathrm{e} 9: 10.0 \mathrm{e} 9$ & $0.001: 0.001$ \\
$10 \mathrm{e} 9: 12 \mathrm{e} 9$ & $0.001: 0.001$ \\
\hline
\end{tabular}

The first guess solution is obtained by comparing the observational CMD with isochrones of different ages and metallicities. We use $N=16$ stellar populations, whose ages, metal content are listed in Table 1.

\subsection{Field star formation history in the HI-shells}

Analogously to Sects. 5.1.1, 5.1.2 we subdivide the shells in two regions. Figure 23 shows the SFR in the shell 37A. As noticed in the cluster age distribution (see Sect. 4) the star formation process has been much more active on the East side of 37A, in the region between the two shells. Concerning the young population, enhancements of the SFR are found at 5, 50, 130 Myr both on the East and on the West side.

Figure 24 presents the SFR in the shell 304A. In the northern side of the supershell the SF was more active at young ages. The SF was continuous from a few Myr to about 160 Myr with period of enhancements from 5 to $40 \mathrm{Myr}$ and between 100-160 Myr. The SFR in both shells is consistent, even if the intensity of the younger episodes was higher in the shell 304A.

Summarizing, in both shells the field star formation was continuous in the past 160 Myr. A global burst of SF is found at
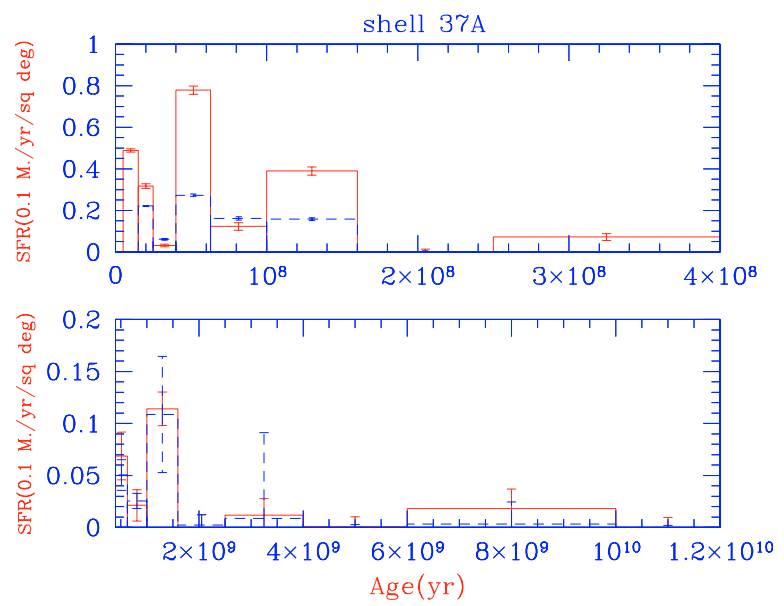

Fig. 23. SFR on the East side of the supershell 37A (solid line) and on the West side (dashed line).
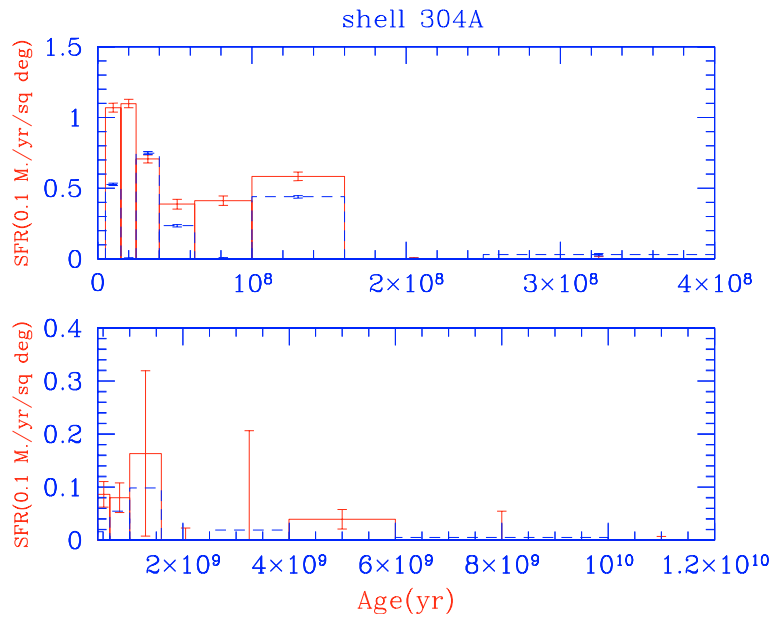

Fig. 24. SFR on the northern side of the supershell 304A (solid line) and on the southern side (dashed line).

ages of a few Myr, which might be responsible of the fractal structure of HI interstellar medium (Stanimirovic et al. 1999; Hatzidimitriou et al. 2005). The SF rate at older ages was less active. Enhancements are found between 100-150 Myr and between 1 and $1.6 \mathrm{Gyr}$, corresponding to a close interaction between SMC and LMC. We remind that the data do not allow precise determination of the SF at older ages.

Comparing with the cluster age distribution (see previous Sections), we find that there is not a complete coincidence between cluster and field star formation, suggesting a different mode of formation. However, formation episodes involving both happened at 5,20 , and finally at $100-150 \mathrm{Myr}$, in coincidence with SMC perigalactic passage.

At present, no detailed studies of the cluster and star formation due to tidal interaction in the SMC are available. However the evolution and star formation history of LMC has been derived in great detail by Bekki \& Chiba (2005). They pointed out that gravitational interactions do not necessarily influence in the same way cluster and field star formation. In fact, cluster formation is expected to take place only if the perturbation due to tidal effects is strong enough to trigger high velocities cloud-cloud collision. Field star formation is more sensitive to tidal triggers and it is expected to take place under less restrictive conditions. The model by Bekki \& Chiba (2005) predicts no cluster formation in the LMC at the time of the first perigalactic 
passage, 6.8 Gyr ago, when field star formation was enhanced, while a strong enhancement of the cluster and field star formation rate in the LMC is expected as a consequence of the strong Galaxy-LMC-SMC interaction between 2-3.5 Gyr ago. At that time, the peak of the cluster formation is almost coincident with the peak of the field star formation, although slightly delayed, taking place $2.5 \mathrm{Gyr}$ ago. Cluster and field star formation is expected in the LMC 100-200 Myr ago, at the time of the most recent collision with the SMC.

Our results point out that the last interaction between SMC and LMC has triggered cluster and field star formation in the $\mathrm{SMC}$, in agreement with what is found in the LMC and is predicted by the models.

\section{Summary and conclusions}

In this paper we revise the cluster and field star formation in the main body of the Small Magellanic Cloud. The main goal is to perform a study of the correlation between young objects and their interstellar environment. The age of 311 clusters and 164 associations is determined through isochrone fitting method. The spatial distribution of the clusters is compared with the HI maps, with the HI velocity dispersion field, with the location of the $\mathrm{CO}$ clouds and with the distribution of young field stars.

Our main results are as follows:

1) The cluster age distribution supports the idea that clusters formed in the last 1 Gyr of the SMC history in a roughly continuous way with periods of enhancement. The age distribution of the clusters in the whole disk presents enhancements, namely between a few Myr and 15 Myr and at 90 Myr. Old objects are mainly located close to the SMC center and on the SW side. Models of the interactions between LMC-SMC and Milky Way predict a close encounter between the MCs roughly 100-200 Myr ago. At that time the star formation is expected to be enhanced not only in the tidal arms, but also in the main body of the SMC. In fact an episode at $90 \mathrm{Myr}$ is found in the age distribution of the clusters that might be due to tidal trigger. However the age distribution presents younger episodes that might have different origin and are possibly due to local phenomena.

2) The two shells $37 \mathrm{~A}$ and $304 \mathrm{~A}$ are clearly visible in the spatial age distribution of the clusters younger than 15 Myr about: the mechanism responsible of the shell formation ( $\mathrm{SN}$, stellar winds, and/or turbulence) is closely related to the cluster formation. The regions have been very active especially at the edges of the shells and in the inter-shell region since $1 \mathrm{Gyr}$ ago. In the supershell 37A clusters younger than a few $10^{7} \mathrm{yr}$ are located at the eastern rim of the supershell $37 \mathrm{~A}$ where gas and dust are located, while older episodes are widely distributed. The cluster age distribution at the eastern part shows a young episode at a few Myr, and several enhancements, namely between a few Myr and $15 \mathrm{Myr}$, and at $80 \mathrm{Myr}$. On the western side, the star formation was less efficient at ages younger than $15 \mathrm{Myr}$, while it was comparable at older ages.

The cluster distribution in the supershell 304A shows a continuous formation from a few Myr to $1 \mathrm{Gyr}$. The dominant episode was between a few Myr and 20 Myr. An enhancement is found at $90 \mathrm{Myr}$.

3) We find that star clusters and associations form in clustered distribution. The typical correlation scale of the clusters is of the order of $500 \mathrm{pc}$, comparable with the dimensions of the large molecular cloud complexes found in the LMC and in the SMC. The two point autocorrelation function of the young massive field stars shows a stronger correlation, but on a comparable scale.

4) A tight cross-correlation between young clusters and the HI intensity is found. The degree of correlation is decreasing with the age of the clusters. Finally clusters older than $300 \mathrm{Myr}$ are located away from the HI peaks. Clusters and associations younger than $10 \mathrm{Myr}$ are related to the $\mathrm{CO}$ clouds in the SW region of the disk, but not in the NE where smaller clouds are found. Older generation is more evenly distributed. This is in agreement with the evolutionary time scale of the giant molecular clouds that is found to be of the order of 10 Myr. This correlation indicates that the molecular gas content is associated to the field and cluster formation, but that its presence does not necessarily imply star formation.

5) A weak relation between the location of the young clusters and the velocity dispersion field of the atomic gas is derived. The shell 304A (where a positive correlation is found) is coincident with a high velocity dispersion region where shocks among clouds might have triggered cluster formation. However this mechanism cannot account for the majority of young objects in the southern shell, 37A where young clusters are located in region of intermediate velocity dispersion.

6) The field star formation was continuous in the past $160 \mathrm{Myr}$. Then periods of quiescence were followed by enhanced activity. A global burst of SF is found at ages of a few Myr, which might be responsible of the fractal structure of $\mathrm{HI}$ interstellar medium. Enhancements are found between 100-150 Myr and between 1 and 1.6 Gyr, corresponding to a close interaction between SMC and LMC. The last tidal interaction between the MCs (100-200 Myr ago) has triggered the formation of both clusters and field stars. However, clusters and field formation rates are not completely coincident, suggesting a different mode of formation.

Acknowledgements. Many thanks are due to G. Bertelli for many helpfull discussions. This work has been partially supported by INAF PRIN 2002.

\section{References}

Bekki, K., \& Chiba, M. 2005, MNRAS, 356, 680

Bekki, K., Couch, W. J., Beasley, M. A., et al. 2004, ApJ, 610, L93 Bertelli, G., Mateo, M., Chiosi, C., \& Bressan, A. 1992, ApJ, 388, 400 Bica, E., \& Dutra, C. M. 2000, AJ, 119, 1214

Bica, E. L. D., \& Schmitt, H. R. 1995, ApJS, 101, 41

Boutloukos, S. G., \& Lamers, H. J. G. L. M. 2003, MNRAS, 338, 717

Caputo, F., Marconi, M., \& Ripepi, V. 1999, ApJ, 525, 784

Chabrier, G. 2003, PASP, 115, 763

Cioni, M.-R. L., Habing, H. J., \& Israel, F. P. 2000, A\&A, 358, L9

Crowl, H. H., Sarajedini, A., Piatti, A. E., et al. 2001, AJ, 122, 220

Da Costa, G. S., \& Hatzidimitriou, D. 1998, AJ, 115, 1934

de Grijs, R., \& Anders, P. 2006, MNRAS, 366, 295

de La Fuente Marcos, R. 1997, A\&A, 322, 764

Dib, S., \& Burkert, A. 2005, ApJ, 630, 238

Dolphin, A. E. 2000, MNRAS, 313, 281

Dolphin, A. E., Walker, A. R., Hodge, P. W., et al. 2001, ApJ, 562, 303

Elmegreen, B. G., \& Efremov, Y. N. 1997, ApJ, 480, 235

Elson, R. A. W., Gilmore, G. F., \& Santiago, B. X. 1997, MNRAS, 289, 157

Fukui, Y. 2005, in The Dusty and Molecular Universe: A Prelude to Herschel and ALMA, $81-86$

Fukui, Y., Mizuno, N., Yamaguchi, R., et al. 1999, PASJ, 51, 745

Gallagher, J. S., Mould, J. R., de Feijter, E., et al. 1996, ApJ, 466, 732

Gardiner, L. T., \& Hatzidimitriou, D. 1992, MNRAS, 257, 195

Gardiner, L. T., Sawa, T., \& Fujimoto, M. 1994, MNRAS, 266, 567

Geha, M. C., Holtzman, J. A., Mould, J. R., et al. 1998, AJ, 115, 1045

Girardi, L., Bressan, A., Chiosi, C., Bertelli, G., \& Nasi, E. 1996, A\&AS, 117, 113

Girardi, L., Bressan, A., Bertelli, G., \& Chiosi, C. 2000, VizieR Online Data Catalog, 414, 10371

Girardi, L., Bertelli, G., Bressan, A., et al. 2002, A\&A, 391, 195

Groenewegen, M. A. T. 2000, A\&A, 363, 901

Harris, H. C. 1981, AJ, 86, 1192 
Harris, J., \& Zaritsky, D. 1999, AJ, 117, 2831

Harris, J., \& Zaritsky, D. 2004, AJ, 127, 1531

Hatzidimitriou, D., Stanimirovic, S., Maragoudaki, F., et al. 2005, MNRAS, 360, 1171 Hodge, P. 1986, PASP, 98, 1113

Holtzman, J. A., Mould, J. R., Gallagher, J. S., et al. 1997, AJ, 113, 656

Hunter, D. A., Elmegreen, B. G., Dupuy, T. J., \& Mortonson, M. 2003, AJ, 126, 1836

Israel, F. P., de Graauw, T., Johansson, L. E. B., et al. 2003, A\&A, 401, 99

Javiel, S. C., Santiago, B. X., \& Kerber, L. O. 2005, A\&A, 431, 73

Kroupa, P., \& Boily, C. M. 2002, MNRAS, 336, 1188

Kumai, Y., Basu, B., \& Fujimoto, M. 1993, ApJ, 404, 144

Lah, P., Kiss, L. L., \& Bedding, T. R. 2005, MNRAS, 359, L42

Lamers, H. J. G. L. M., Anders, P., \& de Grijs, R. 2006, ArXiv Astrophysics e-prints

Lin, D. N. C., Jones, B. F., \& Klemola, A. R. 1995, ApJ, 439, 652

Maragoudaki, F., Kontizas, M., Morgan, D. H., et al. 2001, A\&A, 379, 864

Massey, P. 2002, VizieR Online Data Catalog, 2236, 0

Mathewson, D. S., Ford, V. L., \& Visvanathan, N. 1986, ApJ, 301, 664

McCray, R., \& Kafatos, M. 1987, ApJ, 317, 190

Mizuno, N., Rubio, M., Mizuno, A., et al. 2001, PASJ, 53, L45

Olsen, K. A. G. 1999, AJ, 117, 2244

Olszewski, E. W., Suntzeff, N. B., \& Mateo, M. 1996, ARA\&A, 34, 511

Pagel, B. E. J., \& Tautvaišienè, G. 1999, Ap\&SS, 265, 461

Peebles, P. J. E. 1980, The large-scale structure of the universe, Research supported by the

National Science Foundation (Princeton, N.J.: Princeton University Press), 435

Piatti, A. E., Santos, J. F. C., Clariá, J. J., et al. 2001, MNRAS, 325, 792

Piatti, A. E., Sarajedini, A., Geisler, D., Seguel, J., \& Clark, D. 2005, MNRAS, 358, 1215

Pietrzynski, G., \& Udalski, A. 1999, Acta Astron., 49, 157
Rafelski, M., \& Zaritsky, D 2005, AJ, 129, 2701

Rich, R. M., Shara, M., Fall, S. M., \& Zurek, D. 2000, AJ, 119, 197

Rizzi, L. 2003, Ph.D. Thesis

Rizzi, L., Held, E. V., Bertelli, G., et al. 2002, in Observed HR Diagrams and Stellar Evolution, ASP Conf. Ser., 274, 490

Rubio, M., Lequeux, J., \& Boulanger, F. 1993, A\&A, 271, 9

Sandage, A., Bell, R. A., \& Tripicco, M. J. 1999, ApJ, 522, 250

Smith, H. A., Silbermann, N. A., Baird, S. R., \& Graham, J. A. 1992, AJ, 104, 1430

Stanimirović, S., Staveley-Smith, L., \& Jones, P. A. 2004, ApJ, 604, 176

Stanimirovic, S., Staveley-Smith, L., Dickey, J. M., Sault, R. J., \& Snowden, S. L. 1999, MNRAS, 302, 417

Staveley-Smith, L., Sault, R. J., Hatzidimitriou, D., Kesteven, M. J., \& McConnell, D. 1997, MNRAS, 289, 225

Storm, J., Carney, B. W., Gieren, W. P., et al. 2004, A\&A, 415, 531

Stryker, L. L., Da Costa, G. S., \& Mould, J. R. 1985, ApJ, 298, 544

Tumlinson, J., Shull, J. M., Rachford, B. L., et al. 2002, ApJ, 566, 857

Udalski, A., Szymanski, M., Kubiak, M., et al. 1998, Acta Astron., 48, 147

Vallenari, A., Chiosi, C., Bertelli, G., \& Ortolani, S. 1996, A\&A, 309, 358

Weidner, C., \& Kroupa, P. 2004, MNRAS, 348, 187

Welch, D. L., McLaren, R. A., Madore, B. F., \& McAlary, C. W. 1987, ApJ, 321, 162

Weldrake, D. T. F., Sackett, P. D., Bridges, T. J., \& Freeman, K. C. 2004, AJ, 128, 736

Wyse, R. F. G., Gilmore, G., Houdashelt, M. L., et al. 2002, New Astron., 7, 395

Yoshizawa, A. M., \& Noguchi, M. 2003, MNRAS, 339, 1135

Zaritsky, D., Harris, J., Grebel, E. K., \& Thompson, I. B. 2000, ApJ, 534, L53

Zaritsky, D., Harris, J., Thompson, I. B., Grebel, E. K., \& Massey, P. 2002, AJ, 123, 855

Zhang, Q., Fall, S. M., \& Whitmore, B. C. 2001, ApJ, 561, 727 
E. Chiosi et al.: Cluster and field SFR in the SMC, Online Material $p 1$

\section{Online Material}


E. Chiosi et al.: Cluster and field SFR in the SMC, Online Material p 2

Table 2. Catalog of positions and ages of SMC clusters. Column 1: identification number, Cols. 2 and 3: coordinates, Col. 4: Name, Col. 5: Radius, Col. 8: Type are from Bica \& Dutra (2000). Column 9: Class gives an estimate of the uncertainties on the age determination. Class 1 indicates objects having $\Delta(\log (\operatorname{Age}(\mathrm{yr})))<0.3$; class 2 indicates objects having $0.3<\Delta(\log (\operatorname{Age}(\mathrm{yr})))<0.5$; class 3 indicates objects having $\Delta(\log (\operatorname{Age}(\mathrm{yr})))>0.5$.

\begin{tabular}{|c|c|c|c|c|c|c|c|c|}
\hline 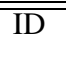 & $\begin{array}{c}\alpha(2000) \\
(\mathrm{deg})\end{array}$ & $\begin{array}{c}\delta(2000) \\
(\mathrm{deg})\end{array}$ & Name & $\begin{array}{c}\begin{array}{c}\text { Radius } \\
\text { (deg) }\end{array}\end{array}$ & $\begin{array}{c}\log (\text { Age }) \\
(y r)\end{array}$ & $\overline{E E(V-I)}$ & Type & 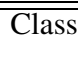 \\
\hline 60 & 9.0583 & -73.4117 & H86-30 & 0.003 & 8.9 & 0.04 & $\mathrm{C}$ & 1 \\
\hline 65 & 9.1583 & -73.0858 & H86-35,SMC_OGLE1 & 0.003 & 8.8 & 0.04 & $\mathrm{C}$ & 1 \\
\hline 66 & 9.2083 & -73.0711 & H86-36 & 0.007 & 8.8 & 0.10 & $\mathrm{AC}$ & 1 \\
\hline 70 & 9.3500 & -73.0306 & H86-38 & 0.010 & 7.6 & 0.04 & A & 1 \\
\hline 72 & 9.3875 & -73.6119 & HW11,SMC_OGLE2 & 0.010 & 8.4 & 0.04 & $\mathrm{C}$ & 1 \\
\hline 73 & 9.3917 & -73.0250 & BS10 & 0.005 & 9.0 & 0.04 & $\mathrm{C}$ & 1 \\
\hline 74 & 9.4208 & -73.1211 & H86-42 & 0.004 & 8.9 & 0.00 & $\mathrm{C}$ & 1 \\
\hline 75 & 9.4250 & -73.9083 & L19,SMC_OGLE3 & 0.014 & 8.9 & 0.04 & $\mathrm{C}$ & 1 \\
\hline 76 & 9.4333 & -73.2111 & B10,SMC_OGLE4 & 0.007 & 9.0 & 0.04 & $\mathrm{C}$ & 3 \\
\hline 77 & 9.4542 & -73.4839 & H86-40 & 0.004 & 8.8 & 0.04 & $\mathrm{C}$ & 1 \\
\hline 78 & 9.4625 & -73.1211 & H86-43 & 0.005 & 8.8 & 0.04 & $\mathrm{C}$ & 1 \\
\hline 79 & 9.5917 & -73.2750 & B12 & 0.004 & 9.0 & 0.04 & $\mathrm{C}$ & 1 \\
\hline 80 & 9.6000 & -73.3783 & BS11 & 0.010 & 7.4 & 0.04 & A & 1 \\
\hline 81 & 9.6542 & -73.8058 & B14,SMC_OGLE162 & 0.005 & 7.9 & 0.04 & $\mathrm{C}$ & 2 \\
\hline 82 & 9.7042 & -73.2756 & H86-47 & 0.002 & 9.0 & 0.04 & $\mathrm{C}$ & 1 \\
\hline 83 & 9.7125 & -73.3742 & HW12,SMC_OGLE163 & 0.007 & 8.7 & 0.30 & $\mathrm{C}$ & 2 \\
\hline 84 & 9.7333 & -73.4089 & H86-48,SMC_OGLE164 & 0.004 & 8.0 & 0.08 & $\mathrm{C}$ & 2 \\
\hline 86 & 9.7458 & -73.9469 & B16 & 0.002 & 9.0 & 0.04 & $\mathrm{C}$ & 1 \\
\hline 87 & 9.7583 & -73.9347 & BS12 & 0.003 & 9.0 & 0.04 & $\mathrm{C}$ & 1 \\
\hline 90 & 9.8417 & -73.2578 & SMC_OGLE5 & 0.007 & 9.0 & 0.01 & $\mathrm{CA}$ & 1 \\
\hline 91 & 9.8583 & -73.3831 & HW12A & 0.005 & 9.0 & 0.04 & $\mathrm{C}$ & 3 \\
\hline 92 & 9.8583 & -73.1064 & H86-55,SMC_OGLE167 & 0.004 & 8.8 & 0.20 & $\mathrm{C}$ & 1 \\
\hline 93 & 9.8792 & -73.4239 & HW13,SMC_OGLE168 & 0.006 & 9.0 & 0.04 & $\mathrm{C}$ & 1 \\
\hline 94 & 9.8958 & -73.3828 & H86-54 & 0.004 & 8.6 & 0.00 & $\mathrm{C}$ & 1 \\
\hline 104 & 10.1125 & -73.4611 & H86-59 & 0.004 & 8.0 & 0.08 & $\mathrm{C}$ & 3 \\
\hline 105 & 10.1250 & -73.2306 & SMC_OGLE7 & 0.008 & 8.3 & 0.15 & $\mathrm{CA}$ & 1 \\
\hline 106 & 10.1292 & -73.4028 & NGC220,K18,L22,ESO29SC3 & 0.010 & 8.1 & 0.08 & $\mathrm{C}$ & 2 \\
\hline 109 & 10.1792 & -73.1181 & H86-60 & 0.003 & 9.0 & 0.10 & $\mathrm{C}$ & 3 \\
\hline 111 & 10.1875 & -73.7406 & B26,SMC_OGLE169 & 0.007 & 8.0 & 0.08 & $\mathrm{C}$ & 2 \\
\hline 113 & 10.1833 & -73.3833 & NGC222,K19,L24,ESO29SC4 & 0.010 & 8.0 & 0.10 & $\mathrm{C}$ & 1 \\
\hline 114 & 10.1875 & -73.3783 & $\mathrm{H}-\mathrm{A} 3$ & 0.065 & 6.6 & 0.04 & A & 1 \\
\hline 118 & 10.2292 & -73.4019 & B23,SMC_OGLE170 & 0.005 & 8.0 & 0.08 & $\mathrm{C}$ & 1 \\
\hline 120 & 10.2500 & -73.6061 & SMC-DEM5 & 0.024 & 7.0 & 0.04 & NA & 1 \\
\hline 122 & 10.2750 & -73.3519 & NGC231,K20,L25,ESO29SC5 & 0.015 & 7.8 & 0.10 & $\mathrm{C}$ & 1 \\
\hline 126 & 10.3375 & -73.3419 & BS15 & 0.020 & 7.8 & 0.10 & A & 1 \\
\hline 128 & 10.4500 & -73.3908 & SMC_OGLE172 & 0.002 & 9.0 & 0.08 & $\mathrm{C}$ & 1 \\
\hline 129 & 10.4833 & -73.4878 & SMC_OGLE166 & 0.005 & 8.5 & 0.10 & $\mathrm{C}$ & 1 \\
\hline 134 & 10.5792 & -73.7419 & SMC-DEM7 & 0.008 & 7.2 & 0.08 & NA & 1 \\
\hline 135 & 10.5917 & -73.7342 & HW16,SMC_OGLE13 & 0.005 & 7.7 & 0.00 & $\mathrm{CN}$ & 2 \\
\hline 136 & 10.6167 & -73.5472 & SMC_OGLE14 & 0.007 & 8.5 & 0.20 & $\mathrm{AC}$ & 1 \\
\hline 138 & 10.7125 & -73.1756 & BS249 & 0.004 & 8.8 & 0.20 & CA & 1 \\
\hline 139 & 10.7250 & -73.2936 & SMC_OGLE15 & 0.008 & 8.1 & 0.08 & CA & 3 \\
\hline 140 & 10.7500 & -73.2944 & $\mathrm{H}-\mathrm{A} 4$ & 0.020 & 7.0 & 0.20 & A & 1 \\
\hline 143 & 10.7417 & -73.1686 & BS16,SMC_OGLE16 & 0.005 & 8.6 & 0.20 & $\mathrm{C}$ & 1 \\
\hline 144 & 10.7583 & -73.1519 & H-A5 & 0.030 & 6.8 & 0.20 & A & 1 \\
\hline 145 & 10.7542 & -73.2578 & BS18 & 0.010 & 7.4 & 0.20 & A & 1 \\
\hline 147 & 10.8083 & -73.0119 & BS17,SMC_OGLE174 & 0.007 & 8.9 & 0.08 & CA & 1 \\
\hline 148 & 10.8125 & -73.2514 & BS250 & 0.005 & 8.8 & 0.08 & $\mathrm{AC}$ & 1 \\
\hline 149 & 10.8667 & -73.3197 & BS19 & 0.033 & 6.8 & 0.20 & A & 3 \\
\hline 150 & 10.8875 & -73.4403 & NGC241,K22w,L29W & 0.008 & 8.3 & 0.10 & $\mathrm{C}$ & 1 \\
\hline 152 & 10.9083 & -73.4436 & NGC242,K22e,L29e & 0.006 & 7.8 & 0.08 & $\mathrm{C}$ & 3 \\
\hline 153 & 10.9083 & -72.9586 & B31,SMC_OGLE19,SMC_OGLE175 & 0.004 & 8.5 & 0.10 & $\mathrm{C}$ & 1 \\
\hline 155 & 10.9083 & -72.9800 & BS20,SMC_OGLE20 & 0.004 & 8.8 & 0.10 & $\mathrm{C}$ & 1 \\
\hline 156 & 10.9083 & -73.2442 & H-A6 & 0.033 & 7.4 & 0.08 & A & 1 \\
\hline 157 & 10.9333 & -72.9767 & H86-70,SMC_OGLE21 & 0.005 & 8.8 & 0.00 & $\mathrm{C}$ & 1 \\
\hline 160 & 10.9917 & -73.1522 & SMC_OGLE22 & 0.006 & 8.6 & 0.20 & CA & 1 \\
\hline 161 & 11.0542 & -73.4506 & SMC-DEM10 & 0.040 & 7.2 & 0.20 & NA & 1 \\
\hline 162 & 11.0542 & -73.6189 & B33,SMC_OGLE23 & 0.004 & 8.4 & 0.08 & $\mathrm{C}$ & 1 \\
\hline 163 & 11.0958 & -72.9456 & B34A & 0.006 & 9.0 & 0.08 & CA & 3 \\
\hline 164 & 11.0958 & -73.2125 & BS23 & 0.020 & 7.2 & 0.10 & A & 1 \\
\hline 165 & 11.1000 & -73.2517 & BS24 & 0.020 & 6.6 & 0.20 & A & 1 \\
\hline
\end{tabular}


Table 2. continued.

\begin{tabular}{|c|c|c|c|c|c|c|c|c|}
\hline ID & $\begin{array}{c}\begin{array}{c}\alpha(2000) \\
(\operatorname{deg})\end{array}\end{array}$ & $\begin{array}{c}\delta(2000) \\
(\mathrm{deg})\end{array}$ & Name & $\begin{array}{c}\text { Radius } \\
\text { (deg) }\end{array}$ & $\begin{array}{c}\log (\text { Age }) \\
(y r)\end{array}$ & $\overline{\overline{E(V-I)}}$ & Type & Class \\
\hline 166 & 11.1917 & -72.8981 & $\mathrm{BS} 25$ & 0.008 & 8.3 & 0.20 & $\mathrm{CA}$ & 1 \\
\hline 168 & 11.2167 & -73.0019 & B34,SMC_OGLE176 & 0.005 & 8.8 & 0.10 & $\mathrm{C}$ & 1 \\
\hline 170 & 11.2292 & -73.1742 & BS27,SMC_OGLE177 & 0.003 & 8.4 & 0.08 & $\mathrm{C}$ & 3 \\
\hline 173 & 11.2292 & -73.2092 & SMC-DEM13nw & 0.016 & 7.0 & 0.10 & NA & 3 \\
\hline 174 & 11.2333 & -73.1697 & SMC-N10,L61-60,SMC-DEM11 & 0.003 & 8.5 & 0.20 & $\mathrm{NC}$ & 1 \\
\hline 175 & 11.2542 & -72.9214 & SMC_OGLE24 & 0.016 & 7.0 & 0.08 & A & 3 \\
\hline 178 & 11.2917 & -73.2342 & SMC-DEM13se,H-A7 & 0.024 & 7.3 & 0.20 & NA & 1 \\
\hline 179 & 11.2958 & -72.8753 & BS28,SMC_OGLE178 & 0.006 & 8.5 & 0.12 & $\mathrm{CA}$ & 1 \\
\hline 180 & 11.3083 & -73.2192 & H86-74,SMC_OGLE25 & 0.006 & 8.0 & 0.08 & $\mathrm{C}$ & 1 \\
\hline 182 & 11.3292 & -73.2589 & SMC-DEM14,H-A8 & 0.016 & 6.8 & 0.08 & NA & 1 \\
\hline 182 & 11.3292 & -73.2589 & SMC-DEM14,H-A8 & 0.016 & 6.8 & 0.20 & NA & 1 \\
\hline 183 & 11.3375 & -73.0356 & SMC_OGLE179 & 0.004 & 8.4 & 0.20 & $\mathrm{CA}$ & 1 \\
\hline 184 & 11.3500 & -73.3761 & NGC248n,SMC-N13B,L61-67n & 0.006 & 7.2 & 0.20 & NA & 1 \\
\hline 186 & 11.3458 & -72.9286 & SMC_OGLE180 & 0.002 & 9.0 & 0.08 & $\mathrm{C}$ & 1 \\
\hline 187 & 11.3583 & -73.3844 & NGC248s,SMC-N13A,L61-67s & 0.006 & 8.6 & 0.08 & $\mathrm{NC}$ & 2 \\
\hline 189 & 11.3583 & -73.4814 & B39,SMC_OGLE27 & 0.005 & 8.6 & 0.08 & $\mathrm{C}$ & 1 \\
\hline 191 & 11.3667 & -72.8194 & SMC_OGLE28 & 0.003 & 8.8 & 0.08 & $\mathrm{C}$ & 2 \\
\hline 192 & 11.3667 & -72.8858 & SMC_OGLE181 & 0.006 & 8.5 & 0.08 & $\mathrm{CA}$ & 3 \\
\hline 194 & 11.3750 & -73.4850 & BS30 & 0.003 & 8.9 & 0.08 & $\mathrm{C}$ & 2 \\
\hline 197 & 11.3875 & -73.1075 & SMC_OGLE30 & 0.006 & 8.7 & 0.25 & $\mathrm{CA}$ & 1 \\
\hline 198 & 11.3917 & -73.2572 & $\mathrm{~B}-\mathrm{OB} 1$ & 0.030 & 8.2 & 0.08 & AN & 3 \\
\hline 200 & 11.4042 & -73.2094 & SMC-DEM17 & 0.015 & 6.8 & 0.08 & NA & 1 \\
\hline 201 & 11.4083 & -73.0747 & BS37 & 0.005 & 6.8 & 0.08 & NA & 1 \\
\hline 203 & 11.4333 & -72.8431 & B36 & 0.006 & 8.4 & 0.08 & $\mathrm{C}$ & 3 \\
\hline 204 & 11.4625 & -72.8403 & SMC_OGLE31 & 0.005 & 8.8 & 0.08 & $\mathrm{CA}$ & 3 \\
\hline 206 & 11.4750 & -73.1142 & SMC-DEM20 & 0.020 & 7.8 & 0.12 & NA & 3 \\
\hline 207 & 11.4750 & -73.5067 & NGC256,K23,L30,ESO29SC11 & 0.007 & 8.0 & 0.20 & $\mathrm{C}$ & 1 \\
\hline 210 & 11.5083 & -73.0936 & SMC-N12,B-OB3 & 0.060 & 7.0 & 0.12 & NA & 3 \\
\hline 211 & 11.5125 & -73.3006 & SMC-DEM19 & 0.050 & 7.0 & 0.20 & NA & 1 \\
\hline 212 & 11.5208 & -73.4439 & BS31 & 0.023 & 8.2 & 0.08 & AN & 1 \\
\hline 213 & 11.5458 & -72.8175 & H86-80,SMC_OGLE184 & 0.003 & 9.0 & 0.08 & $\mathrm{C}$ & 1 \\
\hline 219 & 11.5458 & -73.2733 & BS32 & 0.016 & 6.8 & 0.08 & NA & 1 \\
\hline 223 & 11.5750 & -73.2117 & SMC-DEM22 & 0.015 & 7.0 & 0.08 & NA & 1 \\
\hline 224 & 11.5750 & -73.3925 & SMC-N16,MA130 & 0.010 & 7.1 & 0.24 & NA & 1 \\
\hline 225 & 11.5958 & -73.1333 & BS33 & 0.015 & 7.0 & 0.08 & NA & 1 \\
\hline 226 & 11.5958 & -73.2186 & $\mathrm{H}-\mathrm{A} 10$ & 0.030 & 7.0 & 0.15 & AN & 1 \\
\hline 227 & 11.6125 & -73.1047 & SMC-N12A,SMC-DEM23,MA133 & 0.015 & 7.2 & 0.08 & NA & 1 \\
\hline 228 & 11.6333 & -73.0986 & NGC261,B42,ESO29EN12 & 0.010 & 6.8 & 0.08 & NA & 1 \\
\hline 229 & 11.6458 & -72.7422 & L31,SMC_OGLE36 & 0.009 & 8.5 & 0.08 & $\mathrm{C}$ & 3 \\
\hline 230 & 11.6417 & -72.7739 & H86-83,SMC_OGLE35 & 0.006 & 8.4 & 0.08 & $\mathrm{C}$ & 3 \\
\hline 231 & 11.6417 & -72.7656 & H86-84,SMC_OGLE185 & 0.003 & 8.7 & 0.20 & $\mathrm{C}$ & 1 \\
\hline 232 & 11.6583 & -73.5283 & SMC-N17,SMC-DEM25,MA140 & 0.020 & 7.0 & 0.08 & NA & 1 \\
\hline 234 & 11.6708 & -73.0000 & SMC_OGLE37 & 0.007 & 8.5 & 0.08 & $\mathrm{C}$ & 2 \\
\hline 235 & 11.6708 & -73.3639 & SMC-DEM24,MA142 & 0.015 & 6.7 & 0.20 & NA & 1 \\
\hline 238 & 11.6875 & -73.1714 & MA143 & 0.003 & 8.0 & 0.20 & $\mathrm{NC}$ & 1 \\
\hline 239 & 11.7000 & -73.4353 & SMC-DEM29 & 0.070 & 6.8 & 0.08 & NA & 1 \\
\hline 240 & 11.7333 & -73.4236 & H86-85,SMC_OGLE186 & 0.005 & 8.2 & 0.08 & $\mathrm{C}$ & 1 \\
\hline 242 & 11.7417 & -73.3358 & H86-88 & 0.007 & 8.8 & 0.08 & $\mathrm{CA}$ & 3 \\
\hline 243 & 11.7542 & -73.0928 & SMC-DEM27 & 0.020 & 6.8 & 0.20 & NA & 1 \\
\hline 245 & 11.7542 & -73.3931 & H86-86,SMC_OGLE40 & 0.007 & 8.0 & 0.08 & $\mathrm{C}$ & 1 \\
\hline 246 & 11.7750 & -73.2567 & H86-89,SMC_OGLE38 & 0.007 & 8.5 & 0.08 & $\mathrm{C}$ & 3 \\
\hline 247 & 11.7750 & -73.3714 & H86-87,SMC_OGLE187 & 0.007 & 8.6 & 0.08 & $\mathrm{C}$ & 3 \\
\hline 248 & 11.7958 & -73.2119 & H-A11 & 0.030 & 6.7 & 0.14 & A & 1 \\
\hline 249 & 11.8000 & -73.4772 & NGC265,K24,L34,ESO29SC14 & 0.010 & 8.4 & 0.08 & $\mathrm{C}$ & 3 \\
\hline 251 & 11.8208 & -73.1414 & SNR0045-73.4,MA165 & 0.014 & 8.0 & 0.15 & $\mathrm{~N}$ & 3 \\
\hline 252 & 11.8542 & -72.8408 & L33,SMC_OGLE41 & 0.008 & 7.9 & 0.08 & $\mathrm{C}$ & 2 \\
\hline 253 & 11.8500 & -73.2056 & H86-93,MA172,SMC_OGLE188 & 0.003 & 7.6 & 0.08 & $\mathrm{CN}$ & 2 \\
\hline 256 & 11.8708 & -73.0856 & SMC-DEM31 & 0.013 & 8.6 & 0.15 & $\mathrm{~N}$ & 1 \\
\hline 258 & 11.8875 & -73.3736 & SMC-DEM30,MA184 & 0.014 & 7.0 & 0.20 & NA & 1 \\
\hline 260 & 11.9042 & -73.0142 & H86-95 & 0.003 & 9.0 & 0.08 & $\mathrm{CA}$ & 1 \\
\hline 261 & 11.9125 & -73.1375 & SMC-N19,SMC-DEM32,H-A12 & 0.060 & 7.0 & 0.12 & NA & 3 \\
\hline 264 & 11.9292 & -73.3561 & H-A13 & 0.024 & 6.6 & 0.08 & A & 1 \\
\hline
\end{tabular}


Table 2. continued.

\begin{tabular}{|c|c|c|c|c|c|c|c|c|}
\hline ID & $\begin{array}{c}\alpha(2000) \\
(\mathrm{deg})\end{array}$ & $\begin{array}{c}\delta(2000) \\
(\mathrm{deg})\end{array}$ & Name & $\begin{array}{c}\text { Radius } \\
\text { (deg) }\end{array}$ & $\begin{array}{c}\log (\text { Age }) \\
(y r)\end{array}$ & $\overline{\overline{E(V-I)}}$ & Type & Class \\
\hline 265 & 11.9458 & -73.3031 & SMC-DEM34,MA187 & 0.005 & 7.0 & 0.08 & NA & 1 \\
\hline 268 & 11.9583 & -73.4783 & BS35,SMC_OGLE42 & 0.006 & 8.8 & 0.08 & $\mathrm{C}$ & 1 \\
\hline 269 & 11.9583 & -73.3883 & H-A14 & 0.024 & 7.3 & 0.10 & AN & 3 \\
\hline 270 & 11.9667 & -73.2222 & H86-97,SMC_OGLE43 & 0.006 & 8.5 & 0.08 & $\mathrm{C}$ & 3 \\
\hline 272 & 11.9708 & -73.5314 & H86-94 & 0.005 & 8.7 & 0.08 & $\mathrm{C}$ & 2 \\
\hline 273 & 11.9792 & -72.9556 & H86-98,SMC_OGLE44 & 0.007 & 8.8 & 0.08 & $\mathrm{CA}$ & 3 \\
\hline 274 & 11.9833 & -73.1025 & MA199 & 0.002 & 7.2 & 0.08 & $\mathrm{NC}$ & 3 \\
\hline 275 & 11.9917 & -73.2944 & SMC-N23,SMC-DEM36,MA202 & 0.007 & 8.0 & 0.08 & NA & 1 \\
\hline 276 & 12.0042 & -73.4861 & K25,L35,SMC_OGLE45 & 0.010 & 8.4 & 0.08 & $\mathrm{C}$ & 3 \\
\hline 277 & 12.0083 & -73.2739 & NGC267,SMC-N22,SMC-DEM37 & 0.017 & 7.0 & 0.15 & NA & 1 \\
\hline 283 & 12.0417 & -73.3308 & SMC-N24,SMC-DEM42w,MA210 & 0.010 & 6.8 & 0.08 & NA & 1 \\
\hline 284 & 12.0542 & -72.7931 & H86-99,SMC_OGLE190 & 0.005 & 8.9 & 0.08 & $\mathrm{CA}$ & 3 \\
\hline 286 & 12.0708 & -73.1708 & SMC-DEM39,MA216 & 0.005 & 6.9 & 0.08 & NA & 1 \\
\hline 287 & 12.0708 & -73.4025 & B-OB2 & 0.058 & 6.8 & 0.08 & A & 1 \\
\hline 289 & 12.0833 & -72.7950 & H86-100,SMC_OGLE191 & 0.006 & 8.6 & 0.15 & $\mathrm{CA}$ & 3 \\
\hline 290 & 12.0875 & -73.5303 & NGC269,K26,L37,ESO29SC16 & 0.010 & 8.5 & 0.10 & $\mathrm{C}$ & 3 \\
\hline 294 & 12.1083 & -72.7525 & SMC-DEM46w & 0.033 & 6.7 & 0.08 & NA & 1 \\
\hline 296 & 12.1083 & -73.0072 & SMC_OGLE192 & 0.003 & 8.7 & 0.08 & $\mathrm{C}$ & 3 \\
\hline 297 & 12.1125 & -73.1003 & MA228 & 0.003 & 7.0 & 0.20 & $\mathrm{NC}$ & 3 \\
\hline 298 & 12.1167 & -72.9833 & SMC_OGLE47 & 0.010 & 8.2 & 0.08 & $\mathrm{AC}$ & 3 \\
\hline 299 & 12.1208 & -73.1081 & MA231 & 0.004 & 7.5 & 0.08 & $\mathrm{NC}$ & 3 \\
\hline 301 & 12.1292 & -73.2681 & SMC-N28A,MA234 & 0.007 & 8.2 & 0.08 & NA & 3 \\
\hline 302 & 12.1375 & -73.3069 & B47,SMC_OGLE48 & 0.008 & 8.4 & 0.08 & $\mathrm{C}$ & 3 \\
\hline 303 & 12.1417 & -73.2586 & SMC-N28,SMC-DEM43,MA244 & 0.015 & 7.0 & 0.20 & NA & 3 \\
\hline 304 & 12.1542 & -73.4147 & B48,SMC_OGLE49 & 0.010 & 8.0 & 0.08 & $\mathrm{CA}$ & 3 \\
\hline 305 & 12.1542 & -73.1792 & SMC_OGLE193 & 0.004 & 8.7 & 0.08 & $\mathrm{CA}$ & 3 \\
\hline 307 & 12.1667 & -73.4303 & H-A16 & 0.020 & 6.6 & 0.08 & A & 1 \\
\hline 310 & 12.2167 & -73.5614 & B49 & 0.004 & 9.0 & 0.08 & $\mathrm{C}$ & 1 \\
\hline 311 & 12.2375 & -73.1644 & SMC-N30A,L61-126,MA267 & 0.003 & 7.8 & 0.15 & $\mathrm{NC}$ & 3 \\
\hline 312 & 12.2458 & -73.1511 & SMC_OGLE50 & 0.006 & 7.7 & 0.08 & $\mathrm{NC}$ & 3 \\
\hline 313 & 12.2500 & -73.1389 & SMC-N30,SMC-DEM45 & 0.023 & 7.0 & 0.15 & NA & 1 \\
\hline 314 & 12.2625 & -73.2436 & SNR0047-73.5 & 0.024 & 7.4 & 0.04 & $\mathrm{~N}$ & 3 \\
\hline 315 & 12.2583 & -73.3622 & B50 & 0.005 & 7.0 & 0.08 & $\mathrm{C}$ & 3 \\
\hline 316 & 12.2708 & -73.0511 & H86-103,SMC_OGLE51 & 0.005 & 7.0 & 0.08 & $\mathrm{C}$ & 3 \\
\hline 317 & 12.2750 & -73.3528 & BS41,SMC_OḠLE194 & 0.005 & 8.1 & 0.08 & $\mathrm{C}$ & 1 \\
\hline 318 & 12.3000 & -73.1086 & H86-104,SMC_OGLE52 & 0.003 & 9.0 & 0.08 & $\mathrm{C}$ & 1 \\
\hline 319 & 12.3125 & -72.8800 & SMC-DEM47,B-OB6w & 0.035 & 6.9 & 0.15 & NA & 1 \\
\hline 320 & 12.3167 & -73.2492 & BS42,SMC_OGLE195 & 0.008 & 7.0 & 0.20 & $\mathrm{CA}$ & 1 \\
\hline 321 & 12.3125 & -73.3708 & BS43 & 0.009 & 6.8 & 0.08 & A & 2 \\
\hline 322 & 12.3250 & -73.3722 & L39,SMC_OGLE54 & 0.006 & 8.0 & 0.08 & $\mathrm{C}$ & 2 \\
\hline 323 & 12.3250 & -73.2117 & SMC_OGLE53 & 0.008 & 7.3 & 0.15 & $\mathrm{C}$ & 3 \\
\hline 324 & 12.3375 & -73.1839 & SMC_OGLE55 & 0.005 & 8.0 & 0.20 & $\mathrm{C}$ & 2 \\
\hline 325 & 12.3458 & -73.5303 & H86-102 & 0.005 & 8.3 & 0.10 & $\mathrm{C}$ & 2 \\
\hline 326 & 12.3625 & -73.3986 & SMC_OGLE196 & 0.003 & 8.1 & 0.08 & $\mathrm{C}$ & 3 \\
\hline 327 & 12.3625 & -72.8114 & SMC-DEM46e & 0.032 & 6.8 & 0.20 & NA & 1 \\
\hline 328 & 12.3625 & -73.4306 & SMC-DEM44 & 0.056 & 7.0 & 0.08 & NA & 1 \\
\hline 331 & 12.4000 & -73.2639 & SMC-DEM49 & 0.035 & 6.8 & 0.20 & NA & 1 \\
\hline 332 & 12.4000 & -72.8369 & SMC_OGLE56 & 0.007 & 7.6 & 0.14 & $\mathrm{CA}$ & 1 \\
\hline 334 & 12.4167 & -73.0589 & B52,SMC_OGLE57 & 0.009 & 8.5 & 0.08 & $\mathrm{C}$ & 3 \\
\hline 336 & 12.4208 & -72.8119 & SMC-N32,L61-137,H86-111 & 0.003 & 8.7 & 0.20 & $\mathrm{NC}$ & 3 \\
\hline 337 & 12.4250 & -73.1769 & MA317 & 0.002 & 7.8 & 0.08 & $\mathrm{NC}$ & 2 \\
\hline 338 & 12.4333 & -72.8539 & H86-110 & 0.006 & 7.3 & 0.18 & $\mathrm{CA}$ & 2 \\
\hline 339 & 12.4375 & -72.8661 & H86-109,SMC_OGLE58 & 0.004 & 8.4 & 0.15 & $\mathrm{C}$ & 1 \\
\hline 340 & 12.4417 & -73.1736 & SMC-N34,L61-142,SMC-DEM50 & 0.005 & 7.5 & 0.08 & $\mathrm{NC}$ & 3 \\
\hline 341 & 12.4500 & -73.0978 & BS44 & 0.024 & 6.9 & 0.20 & A & 1 \\
\hline 344 & 12.5000 & -73.2550 & H86-107,SMC_OGLE61 & 0.010 & 7.8 & 0.15 & $\mathrm{CA}$ & 1 \\
\hline 346 & 12.5167 & -73.0528 & SMC-DEM51 & 0.038 & 6.8 & 0.60 & NA & 1 \\
\hline 347 & 12.5167 & -73.3367 & H-A17 & 0.025 & 7.0 & 0.20 & A & 1 \\
\hline 348 & 12.5167 & -73.3844 & B53,SMC_OGLE197 & 0.008 & 8.2 & 0.08 & $\mathrm{C}$ & 1 \\
\hline 349 & 12.5417 & -73.1731 & H-A18 & 0.023 & 6.8 & 0.20 & A & 1 \\
\hline 350 & 12.5333 & -73.1906 & H86-112,SMC_OGLE198 & 0.005 & 8.0 & 0.20 & $\mathrm{C}$ & 2 \\
\hline 352 & 12.5500 & -73.3472 & BS39 & 0.004 & 7.5 & 0.08 & $\mathrm{C}$ & 1 \\
\hline
\end{tabular}


Table 2. continued.

\begin{tabular}{|c|c|c|c|c|c|c|c|c|}
\hline 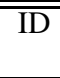 & $\begin{array}{c}(2000) \\
(\operatorname{deg})\end{array}$ & $\begin{array}{c}\delta(2000) \\
(\mathrm{deg})\end{array}$ & Name & $\begin{array}{c}\text { Radius } \\
\text { (deg) }\end{array}$ & $\begin{array}{c}\log (\text { Age }) \\
(y r)\end{array}$ & $\overline{E E(V-I)}$ & Type & Class \\
\hline 354 & 12.5667 & -73.0333 & BS45,SMC_OGLE59 & 0.008 & 7.7 & 0.18 & $\mathrm{CA}$ & 1 \\
\hline 355 & 12.5750 & -73.3247 & MA351 & 0.004 & 7.4 & 0.15 & $\mathrm{NC}$ & 1 \\
\hline 356 & 12.5875 & -72.8811 & SMC-N36w,SMC-DEM54w,H-A19 & 0.032 & 6.9 & 0.15 & NA & 1 \\
\hline 358 & 12.5917 & -73.3878 & B55,SMC_OGLE60 & 0.006 & 8.0 & 0.08 & $\mathrm{C}$ & 2 \\
\hline 359 & 12.6167 & -73.2033 & B54,SMC_OGLE62 & 0.005 & 8.3 & 0.08 & $\mathrm{C}$ & 3 \\
\hline 361 & 12.6292 & -73.3364 & H86-106w & 0.004 & 8.7 & 0.15 & $\mathrm{C}$ & 3 \\
\hline 362 & 12.6417 & -73.3386 & SMC-DEM52,MA370 & 0.010 & 6.8 & 0.15 & NA & 1 \\
\hline 363 & 12.6542 & -73.0578 & H86-115,SMC_OGLE63 & 0.013 & 8.0 & 0.08 & $\mathrm{AC}$ & 1 \\
\hline 364 & 12.6583 & -72.7956 & SMC-N37,SMC-DEM55,H-A23 & 0.050 & 6.8 & 0.15 & NA & 1 \\
\hline 365 & 12.6542 & -73.3364 & H86-106e & 0.005 & 7.1 & 0.15 & $\mathrm{C}$ & 3 \\
\hline 367 & 12.6625 & -72.9789 & BS46,SMC_OGLE200 & 0.004 & 8.0 & 0.15 & $\mathrm{C}$ & 3 \\
\hline 368 & 12.6667 & -72.9653 & H86-116,SMC_OGLE64 & 0.004 & 8.0 & 0.15 & $\mathrm{C}$ & 1 \\
\hline 369 & 12.6708 & -72.9672 & L40,H-A22 & 0.015 & 6.6 & 0.60 & A & 1 \\
\hline 370 & 12.6792 & -72.7819 & MA384 & 0.003 & 6.8 & 0.15 & $\mathrm{NC}$ & 3 \\
\hline 371 & 12.6708 & -73.4628 & $\mathrm{H}-\mathrm{A} 21$ & 0.029 & 7.0 & 0.08 & A & 1 \\
\hline 373 & 12.6917 & -73.2972 & $\mathrm{H}-\mathrm{A} 24$ & 0.020 & 7.0 & 0.15 & A & 1 \\
\hline 374 & 12.6750 & -73.3969 & BS48,SMC_OGLE201 & 0.007 & 8.0 & 0.08 & $\mathrm{AC}$ & 1 \\
\hline 376 & 12.7167 & -72.8728 & SMC-N36e,SMC-DEM54e,B-OB6e & 0.035 & 6.8 & 0.15 & NA & 1 \\
\hline 377 & 12.7333 & -72.7278 & L41,SMC_OGLE67 & 0.005 & 8.1 & 0.15 & $\mathrm{C}$ & 1 \\
\hline 378 & 12.7208 & -73.4061 & H86-108,MA401 & 0.008 & 7.0 & 0.20 & NA & 1 \\
\hline 379 & 12.7292 & -73.0575 & SMC_OGLE65 & 0.005 & 8.2 & 0.12 & $\mathrm{C}$ & 1 \\
\hline 380 & 12.7333 & -72.7531 & BS49 & 0.013 & 6.8 & 0.25 & NA & 1 \\
\hline 381 & 12.7292 & -73.2031 & B56,SMC_OGLE66 & 0.004 & 7.7 & 0.10 & $\mathrm{C}$ & 3 \\
\hline 382 & 12.7333 & -73.2892 & BS40,SMC_OGLE68 & 0.007 & 7.0 & 0.16 & $\mathrm{CA}$ & 3 \\
\hline 383 & 12.7500 & -72.8844 & SMC-N41,L61-166,MA424 & 0.003 & 6.7 & 0.30 & $\mathrm{NC}$ & 3 \\
\hline 384 & 12.7458 & -73.5036 & H86-105,SMC_OGLE202 & 0.004 & 7.9 & 0.08 & $\mathrm{C}$ & 2 \\
\hline 385 & 12.7708 & -73.3922 & SMC-DEM53 & 0.029 & 7.0 & 0.08 & NA & 1 \\
\hline 387 & 12.7958 & -73.3681 & SNR0049-73.6 & 0.016 & 8.2 & 0.08 & $\mathrm{~N}$ & 1 \\
\hline 388 & 12.8083 & -72.6928 & $\mathrm{H}-\mathrm{A} 25$ & 0.050 & 7.0 & 0.15 & NA & 1 \\
\hline 389 & 12.8083 & -73.1614 & NGC290,L42,ESO29SC19 & 0.009 & 7.0 & 0.20 & $\mathrm{C}$ & 3 \\
\hline 390 & 12.7917 & -73.5900 & H86-113,SMC_OGLE203 & 0.003 & 8.1 & 0.15 & $\mathrm{C}$ & 1 \\
\hline 391 & 12.8375 & -73.1386 & H86-121,SMC_OGLE204 & 0.005 & 8.8 & 0.08 & $\mathrm{C}$ & 3 \\
\hline 392 & 12.8333 & -73.5064 & SMC-DEM59 & 0.012 & 6.8 & 0.08 & NA & 1 \\
\hline 393 & 12.8583 & -73.2833 & BS251,SMC_OGLE70 & 0.003 & 8.4 & 0.08 & $\mathrm{CA}$ & 2 \\
\hline 394 & 12.8667 & -73.0867 & H-A27A & 0.026 & 6.8 & 0.15 & A & 1 \\
\hline 395 & 12.8625 & -73.1567 & BS51 & 0.027 & 7.0 & 0.20 & A & 1 \\
\hline 396 & 12.8750 & -73.3356 & BS252 & 0.005 & 7.9 & 0.20 & $\mathrm{AC}$ & 1 \\
\hline 398 & 12.8875 & -72.7942 & B-OB7 & 0.033 & 6.8 & 0.15 & AN & 2 \\
\hline 399 & 12.8833 & -72.9792 & H86-124,SMC_OGLE205 & 0.007 & 8.1 & 0.08 & $\mathrm{C}$ & 1 \\
\hline 400 & 12.9042 & -72.5419 & H86-119 & 0.005 & 7.0 & 0.15 & $\mathrm{C}$ & 3 \\
\hline 401 & 12.8833 & -73.0106 & B57,SMC_OGLE71 & 0.010 & 7.1 & 0.20 & $\mathrm{C}$ & 2 \\
\hline 403 & 12.9250 & -73.2297 & SMC-N45,L61-189,B60 & 0.006 & 6.6 & 0.20 & $\mathrm{NC}$ & 3 \\
\hline 404 & 12.9333 & -72.8403 & B59,L61-183,MA488 & 0.007 & 8.0 & 0.14 & $\mathrm{CN}$ & 1 \\
\hline 408 & 12.9333 & -73.1669 & H86-123,SMC_OGLE206 & 0.005 & 8.3 & 0.08 & $\mathrm{C}$ & 1 \\
\hline 409 & 12.9458 & -72.8464 & SMC-N46,L61-184,SMC-DEM62 & 0.005 & 8.1 & 0.10 & $\mathrm{NC}$ & 3 \\
\hline 411 & 12.9542 & -72.5411 & H86-127,SMC_OGLE207 & 0.005 & 8.4 & 0.08 & $\mathrm{C}$ & 2 \\
\hline 412 & 12.9625 & -72.5478 & B-OB9 & 0.023 & 6.9 & 0.20 & A & 1 \\
\hline 413 & 12.9667 & -72.6786 & SMC-DEM63,B-OB8 & 0.092 & 6.8 & 0.30 & NA & 1 \\
\hline 415 & 12.9708 & -72.9539 & K29,L44,SMC_OGLE74 & 0.008 & 8.1 & 0.08 & $\mathrm{C}$ & 1 \\
\hline 416 & 12.9750 & -73.0981 & H86-126,SMC_OGLE75 & 0.004 & 8.2 & 0.10 & $\mathrm{C}$ & 1 \\
\hline 422 & 13.0125 & -72.8178 & H86-128,H86-131,SMC_OGLE208 & 0.004 & 8.3 & 0.08 & $\mathrm{C}$ & 1 \\
\hline 423 & 13.0125 & -72.6589 & MA524 & 0.025 & 6.7 & 0.15 & NA & 1 \\
\hline 426 & 13.0250 & -73.4450 & SMC-DEM70s & 0.050 & 6.8 & 0.20 & NA & 1 \\
\hline 427 & 13.0333 & -73.3153 & BS54 & 0.005 & 6.7 & 0.18 & $\mathrm{AC}$ & 3 \\
\hline 430 & 13.0500 & -72.5308 & H86-129,SMC_OGLE76 & 0.005 & 8.0 & 0.08 & $\mathrm{C}$ & 1 \\
\hline 431 & 13.0542 & -73.0033 & BS56,SMC_OGLE77 & 0.006 & 8.1 & 0.08 & $\mathrm{C}$ & 1 \\
\hline 432 & 13.0625 & -72.7661 & BS253,SMC_OGLE209 & 0.005 & 7.1 & 0.18 & $\mathrm{AC}$ & 1 \\
\hline 433 & 13.0542 & -73.2986 & SMC-DEM70n & 0.083 & 7.0 & 0.30 & NA & 1 \\
\hline 434 & 13.0708 & -73.3756 & H86-125,SMC_OGLE79 & 0.006 & 8.2 & 0.08 & $\mathrm{CA}$ & 1 \\
\hline 436 & 13.0708 & -73.0178 & H86-130,SMC_OGLE78 & 0.006 & 7.0 & 0.08 & $\mathrm{C}$ & 3 \\
\hline 438 & 13.0875 & -73.1622 & B-OB5ne & 0.027 & 6.8 & 0.18 & A & 1 \\
\hline 439 & 13.0875 & -73.3272 & H-A28 & 0.023 & 7.3 & 0.25 & AN & 1 \\
\hline
\end{tabular}


Table 2. continued.

\begin{tabular}{|c|c|c|c|c|c|c|c|c|}
\hline 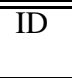 & $\begin{array}{c}\alpha(2000) \\
(\mathrm{deg})\end{array}$ & $\begin{array}{c}\delta(2000) \\
(\mathrm{deg})\end{array}$ & Name & $\begin{array}{c}\text { Radius } \\
\text { (deg) }\end{array}$ & $\begin{array}{c}\log (\text { Age }) \\
(y r)\end{array}$ & $\overline{E E(V-I)}$ & Type & Class \\
\hline 440 & 13.1250 & -73.0497 & B64,SMC_OGLE210 & 0.006 & 8.1 & 0.08 & $\mathrm{C}$ & 1 \\
\hline 441 & 13.1333 & -73.0361 & BS57,SMC_OGLE211 & 0.005 & 7.6 & 0.17 & $\mathrm{C}$ & 1 \\
\hline 442 & 13.1417 & -72.6817 & H86-133,H86-137,SMC_OGLE81 & 0.006 & 7.4 & 0.20 & $\mathrm{C}$ & 1 \\
\hline 444 & 13.1292 & -72.6294 & H86-132,SMC_OGLE80 & 0.004 & 8.4 & 0.08 & $\mathrm{C}$ & 3 \\
\hline 445 & 13.1333 & -73.0514 & H-A29 & 0.022 & 6.7 & 0.28 & A & 1 \\
\hline 448 & 13.1500 & -73.3808 & BS58 & 0.005 & 8.5 & 0.08 & $\mathrm{C}$ & 1 \\
\hline 449 & 13.1667 & -72.6744 & BS59 & 0.023 & 6.8 & 0.25 & NA & 1 \\
\hline 450 & 13.1542 & -73.4378 & SMC-N51,L61-219,SMC-DEM72 & 0.008 & 6.9 & 0.20 & NA & 1 \\
\hline 451 & 13.1750 & -72.9256 & BS60,SMC_OGLE82 & 0.007 & 7.9 & 0.15 & $\mathrm{C}$ & 1 \\
\hline 453 & 13.1833 & -72.9800 & B65,SMC_OGLE83 & 0.006 & 7.8 & 0.08 & $\mathrm{C}$ & 1 \\
\hline 454 & 13.1750 & -73.0008 & H-A30 & 0.017 & 6.9 & 0.50 & A & 1 \\
\hline 455 & 13.1792 & -73.0292 & BS61 & 0.005 & 6.8 & 0.08 & $\mathrm{CA}$ & 3 \\
\hline 456 & 13.1875 & -72.6325 & SMC-N50,SMC-DEM68se & 0.018 & 6.7 & 0.10 & NA & 1 \\
\hline 457 & 13.1917 & -72.7011 & H-A31 & 0.025 & 6.7 & 0.14 & A & 1 \\
\hline 458 & 13.2000 & -72.7961 & B66,SMC_OGLE85 & 0.005 & 8.6 & 0.08 & $\mathrm{C}$ & 1 \\
\hline 459 & 13.2000 & -72.9894 & H86-134e,SMC_OGLE213 & 0.004 & 8.1 & 0.08 & $\mathrm{C}$ & 3 \\
\hline 461 & 13.1958 & -73.4069 & BS63,SMC_OGLE84 & 0.004 & 8.5 & 0.08 & $\mathrm{C}$ & 3 \\
\hline 462 & 13.2083 & -72.7169 & BS64 & 0.008 & 7.4 & 0.13 & $\mathrm{AC}$ & 1 \\
\hline 463 & 13.2042 & -73.4119 & B67,SMC_OGLE87 & 0.005 & 8.5 & 0.20 & $\mathrm{C}$ & 3 \\
\hline 464 & 13.2125 & -72.7353 & BS254 & 0.004 & 7.1 & 0.08 & $\mathrm{C}$ & 1 \\
\hline 466 & 13.2000 & -72.5106 & H86-135,SMC_OGLE86 & 0.005 & 8.7 & 0.08 & $\mathrm{C}$ & 1 \\
\hline 467 & 13.2167 & -73.0292 & BS255 & 0.003 & 8.9 & 0.08 & $\mathrm{C}$ & 1 \\
\hline 468 & 13.2125 & -73.1344 & H-A32 & 0.033 & 6.9 & 0.10 & A & 1 \\
\hline 470 & 13.2542 & -72.8969 & K31,L46,SMC_OGLE88 & 0.023 & 7.2 & 0.20 & $\mathrm{C}$ & 1 \\
\hline 471 & 13.2542 & -73.1872 & BS66 & 0.010 & 7.0 & 0.15 & AN & 3 \\
\hline 472 & 13.2667 & -72.6308 & H-A33 & 0.025 & 6.8 & 0.20 & A & 1 \\
\hline 473 & 13.2792 & -72.6244 & B69,SMC_OGLE89 & 0.005 & 7.7 & 0.08 & $\mathrm{C}$ & 2 \\
\hline 475 & 13.2750 & -73.3803 & NGC294,L47,ESO29SC22 & 0.014 & 8.6 & 0.13 & $\mathrm{C}$ & 1 \\
\hline 476 & 13.2875 & -72.8328 & H86-140,SMC_OGLE214 & 0.004 & 7.8 & 0.08 & $\mathrm{C}$ & 2 \\
\hline 477 & 13.2917 & -72.5736 & H86-138,SMC_OGLE91 & 0.004 & 8.6 & 0.08 & $\mathrm{C}$ & 1 \\
\hline 479 & 13.2958 & -73.2203 & B-OB5se & 0.046 & 8.3 & 0.15 & AN & 3 \\
\hline 480 & 13.3083 & -72.8772 & SMC-DEM69 & 0.040 & 6.8 & 0.25 & NA & 2 \\
\hline 482 & 13.3250 & -72.7667 & B71,SMC_OGLE92 & 0.007 & 7.3 & 0.15 & $\mathrm{C}$ & 1 \\
\hline 486 & 13.3583 & -72.6825 & B72 & 0.010 & 7.4 & 0.08 & $\mathrm{C}$ & 1 \\
\hline 487 & 13.3792 & -72.6678 & H86-143,SMC_OGLE93 & 0.007 & 7.8 & 0.08 & $\mathrm{C}$ & 3 \\
\hline 488 & 13.3833 & -72.5411 & H-A36 & 0.052 & 6.9 & 0.20 & A & 1 \\
\hline 489 & 13.3833 & -73.3508 & BS67 & 0.005 & 8.7 & 0.08 & $\mathrm{AC}$ & 1 \\
\hline 491 & 13.4000 & -72.6417 & BS257 & 0.007 & 8.1 & 0.08 & $\mathrm{AC}$ & 1 \\
\hline 493 & 13.4167 & -72.6597 & SMC-N52A,L61-243 & 0.004 & 7.0 & 0.20 & $\mathrm{NC}$ & 3 \\
\hline 494 & 13.4167 & -72.6831 & H-A35 & 0.028 & 7.0 & 0.15 & AN & 2 \\
\hline 496 & 13.4250 & -72.6542 & SMC-N52B,L61-244,B73 & 0.004 & 7.0 & 0.30 & $\mathrm{NC}$ & 3 \\
\hline 497 & 13.4250 & -73.2442 & SMC-DEM74 & 0.075 & 6.8 & 0.15 & NA & 1 \\
\hline 498 & 13.4250 & -73.3589 & BS68,SMC_OGLE95 & 0.007 & 8.3 & 0.15 & $\mathrm{CA}$ & 2 \\
\hline 499 & 13.4583 & -72.8964 & H86-147,SM̄C_OGLE216 & 0.010 & 8.5 & 0.08 & $\mathrm{C}$ & 2 \\
\hline 501 & 13.4792 & -72.6689 & H86-148 & 0.004 & 8.7 & 0.14 & $\mathrm{C}$ & 1 \\
\hline 503 & 13.4833 & -72.8567 & BS69,SMC_OGLE217 & 0.005 & 8.8 & 0.08 & CA & 3 \\
\hline 505 & 13.4917 & -72.6933 & B75 & 0.010 & 7.4 & 0.12 & $\mathrm{CA}$ & 1 \\
\hline 506 & 13.4917 & -72.7344 & BS71 & 0.016 & 6.7 & 0.20 & NA & 1 \\
\hline 510 & 13.5458 & -72.8650 & BS72,SMC_OGLE97 & 0.006 & 8.8 & 0.08 & $\mathrm{CA}$ & 1 \\
\hline 512 & 13.5625 & -73.2853 & SMC-DEM78 & 0.011 & 6.8 & 0.20 & NA & 1 \\
\hline 513 & 13.5792 & -72.5147 & H86-149 & 0.005 & 8.8 & 0.08 & $\mathrm{C}$ & 1 \\
\hline 515 & 13.5958 & -72.6947 & H86-152,SMC_OGLE218 & 0.007 & 8.4 & 0.22 & $\mathrm{C}$ & 1 \\
\hline 516 & 13.5917 & -73.0456 & H86-151 & 0.004 & 9.0 & 0.08 & $\mathrm{C}$ & 1 \\
\hline 517 & 13.6083 & -72.8847 & BS258 & 0.005 & 8.1 & 0.15 & A & 1 \\
\hline 520 & 13.6375 & -72.7933 & BS73 & 0.035 & 7.0 & 0.26 & NA & 1 \\
\hline 522 & 13.6667 & -72.7153 & SMC-DEM80 & 0.090 & 7.2 & 0.27 & NA & 1 \\
\hline 525 & 13.6958 & -73.2236 & B80,SMC_OGLE98 & 0.009 & 8.7 & 0.08 & $\mathrm{C}$ & 1 \\
\hline 526 & 13.7000 & -72.4661 & B79,SMC_OGLE99 & 0.007 & 7.3 & 0.08 & $\mathrm{C}$ & 1 \\
\hline 527 & 13.7083 & -72.6989 & H86-156 & 0.004 & 8.8 & 0.08 & $\mathrm{C}$ & 1 \\
\hline 528 & 13.7167 & -72.4386 & H-A38 & 0.047 & 7.1 & 0.10 & A & 1 \\
\hline 529 & 13.7167 & -72.9164 & B76,SMC_OGLE219 & 0.003 & 8.4 & 0.08 & $\mathrm{C}$ & 1 \\
\hline 531 & 13.7292 & -72.4419 & BS259 & 0.005 & 7.6 & 0.08 & $\mathrm{C}$ & 1 \\
\hline
\end{tabular}


Table 2. continued.

\begin{tabular}{|c|c|c|c|c|c|c|c|c|}
\hline $\begin{array}{l}\text { ID } \\
\end{array}$ & $\begin{array}{c}\alpha(2000) \\
(\mathrm{deg})\end{array}$ & $\begin{array}{c}\delta(2000) \\
(\mathrm{deg})\end{array}$ & Name & $\begin{array}{c}\text { Radius } \\
\text { (deg) }\end{array}$ & $\begin{array}{c}\log (\text { Age }) \\
(y r)\end{array}$ & $E(V-I)$ & Type & Class \\
\hline 533 & 13.8000 & -72.6833 & H86-159,SMC_OGLE102 & 0.004 & 9.0 & 0.08 & $\mathrm{C}$ & 1 \\
\hline 534 & 13.7875 & -72.8111 & H86-158,H86-161,SMC_OGLE100 & 0.005 & 7.6 & 0.08 & $\mathrm{C}$ & 1 \\
\hline 535 & 13.8000 & -73.2967 & H86-155,SMC_OGLE101 & 0.004 & 8.7 & 0.08 & $\mathrm{C}$ & 1 \\
\hline 537 & 13.8083 & -72.6011 & SMC_OGLE220 & 0.003 & 9.0 & 0.08 & $\mathrm{CA}$ & 1 \\
\hline 541 & 13.8417 & -72.7825 & B-OB12 & 0.038 & 7.6 & 0.15 & AN & 2 \\
\hline 542 & 13.8750 & -73.0714 & B83,SMC_OGLE103 & 0.005 & 8.6 & 0.08 & $\mathrm{C}$ & 1 \\
\hline 544 & 13.8875 & -72.8328 & K34,L53,SMC_OGLE104 & 0.005 & 8.6 & 0.08 & $\mathrm{C}$ & 3 \\
\hline 550 & 13.9292 & -72.8800 & H86-165,H86-168,SMC_OGLE105 & 0.009 & 8.0 & 0.30 & A & 3 \\
\hline 552 & 13.9375 & -72.7050 & H86-164,SMC_OGLE221 & 0.004 & 8.7 & 0.08 & $\mathrm{C}$ & 1 \\
\hline 554 & 13.9500 & -72.7019 & BS262 & 0.010 & 6.7 & 0.25 & A & 1 \\
\hline 557 & 13.9958 & -73.0914 & H86-167 & 0.006 & 8.7 & 0.08 & $\mathrm{C}$ & 1 \\
\hline 564 & 14.0375 & -72.7939 & H86-169 & 0.003 & 7.2 & 0.08 & $\mathrm{C}$ & 3 \\
\hline 566 & 14.0375 & -73.2061 & SMC_OGLE106 & 0.007 & 8.0 & 0.08 & $\mathrm{CA}$ & 1 \\
\hline 568 & 14.0792 & -72.4639 & NGC330,K35,L54,ESO29SC24 & 0.023 & 8.0 & 0.08 & $\mathrm{C}$ & 3 \\
\hline 569 & 14.0708 & -72.7856 & SMC-DEM88 & 0.013 & 7.2 & 0.11 & NA & 1 \\
\hline 570 & 14.0667 & -72.5164 & B86,SMC_OGLE222 & 0.005 & 8.0 & 0.08 & $\mathrm{C}$ & 1 \\
\hline 573 & 14.0917 & -72.4547 & $\mathrm{H}-\mathrm{A} 40$ & 0.059 & 6.7 & 0.20 & A & 2 \\
\hline 574 & 14.1083 & -72.4958 & BS81,SMC_OGLE223 & 0.005 & 8.0 & 0.08 & $\mathrm{C}$ & 3 \\
\hline 575 & 14.1250 & -72.5436 & BS82 & 0.005 & 8.7 & 0.20 & $\mathrm{C}$ & 1 \\
\hline 576 & 14.1250 & -72.8181 & BS264 & 0.014 & 7.0 & 0.20 & NA & 1 \\
\hline 577 & 14.1417 & -72.5022 & H86-172,SMC_OGLE108 & 0.005 & 8.8 & 0.08 & $\mathrm{C}$ & 1 \\
\hline 579 & 14.1667 & -72.4875 & SMC-DEM87 & 0.067 & 6.6 & 0.20 & NA & 1 \\
\hline 583 & 14.2208 & -72.8069 & SMC-DEM89 & 0.027 & 7.0 & 0.20 & NA & 1 \\
\hline 585 & 14.2750 & -72.5456 & BS83 & 0.007 & 6.8 & 0.18 & $\mathrm{AC}$ & 2 \\
\hline 589 & 14.3250 & -72.9336 & H86-174,SMC_OGLE225 & 0.004 & 8.4 & 0.08 & $\mathrm{C}$ & 3 \\
\hline 591 & 14.3375 & -72.5753 & H86-173 & 0.005 & 8.0 & 0.08 & $\mathrm{C}$ & 1 \\
\hline 593 & 14.3792 & -72.2644 & L56,SMC-S26,SMC_OGLE109 & 0.008 & 7.4 & 0.15 & $\mathrm{C}$ & 1 \\
\hline 594 & 14.3750 & -72.5400 & SMC-N61,L61-321,MA1011 & 0.002 & 8.4 & 0.08 & $\mathrm{NC}$ & 2 \\
\hline 597 & 14.4417 & -72.7058 & H86-178,SMC_OGLE110 & 0.005 & 7.5 & 0.08 & $\mathrm{C}$ & 1 \\
\hline 598 & 14.4500 & -72.5394 & BS87 & 0.007 & 8.4 & 0.08 & $\mathrm{AC}$ & 1 \\
\hline 600 & 14.4625 & -72.2239 & BS266 & 0.020 & 7.0 & 0.10 & A & 1 \\
\hline 601 & 14.4583 & -72.9436 & BS88,SMC_OGLE111 & 0.005 & 8.9 & 0.08 & $\mathrm{C}$ & 1 \\
\hline 603 & 14.4583 & -72.4400 & H86-175,SMC_OGLE227 & 0.003 & 8.0 & 0.08 & $\mathrm{C}$ & 1 \\
\hline 604 & 14.4583 & -72.5081 & H86-177,SMC_OGLE226 & 0.006 & 8.2 & 0.08 & $\mathrm{C}$ & 3 \\
\hline 605 & 14.4708 & -72.4967 & H86-176 & 0.005 & 8.0 & 0.08 & $\mathrm{C}$ & 3 \\
\hline 607 & 14.4792 & -72.2911 & B90 & 0.004 & 8.2 & 0.08 & $\mathrm{C}$ & 2 \\
\hline 609 & 14.4833 & -72.6572 & SMC-N62,SMC-DEM93 & 0.010 & 7.2 & 0.20 & NA & 1 \\
\hline 610 & 14.4958 & -72.2344 & BS267 & 0.005 & 7.9 & 0.08 & $\mathrm{AC}$ & 1 \\
\hline 612 & 14.4917 & -72.5483 & B-OB13 & 0.075 & 6.8 & 0.14 & A & 1 \\
\hline 614 & 14.5292 & -72.2906 & $\mathrm{H}-\mathrm{A} 43$ & 0.028 & 6.9 & 0.15 & AN & 2 \\
\hline 615 & 14.5292 & -72.6606 & H-A42 & 0.032 & 7.1 & 0.15 & AN & 2 \\
\hline 616 & 14.5333 & -72.5983 & $\mathrm{H}-\mathrm{A} 41$ & 0.004 & 7.0 & 0.08 & A & 1 \\
\hline 624 & 14.5792 & -72.2992 & H86-181,SMC_OGLE228 & 0.005 & 7.8 & 0.08 & $\mathrm{C}$ & 2 \\
\hline 625 & 14.5875 & -72.3128 & BS268 & 0.047 & 6.9 & 0.18 & NA & 1 \\
\hline 626 & 14.5958 & -72.2119 & BS270 & 0.005 & 8.0 & 0.08 & $\mathrm{CA}$ & 1 \\
\hline 630 & 14.6083 & -72.6658 & SMC-N64A,L61-335 & 0.006 & 6.9 & 0.12 & $\mathrm{NC}$ & 3 \\
\hline 632 & 14.6417 & -72.2367 & SMC-DEM98 & 0.016 & 7.2 & 0.13 & NA & 1 \\
\hline 633 & 14.6417 & -72.2811 & H86-183,SMC_OGLE115 & 0.005 & 8.0 & 0.08 & $\mathrm{C}$ & 2 \\
\hline 634 & 14.6542 & -72.2242 & BS271 & 0.005 & 7.1 & 0.20 & $\mathrm{NC}$ & 3 \\
\hline 635 & 14.6583 & -72.2344 & BS272,SMC_OGLE229 & 0.005 & 7.2 & 0.17 & $\mathrm{NC}$ & 1 \\
\hline 638 & 14.7292 & -72.2444 & SMC-DEM99 & 0.010 & 7.0 & 0.10 & NA & 1 \\
\hline 639 & 14.7292 & -72.8303 & H86-185 & 0.003 & 8.7 & 0.08 & $\mathrm{C}$ & 3 \\
\hline 640 & 14.7708 & -72.1769 & NGC346,SMC-N66,K39,L60 & 0.070 & 7.2 & 0.14 & NA & 1 \\
\hline 641 & 14.7708 & -72.7867 & SMC_OGLE116 & 0.008 & 8.0 & 0.08 & $\mathrm{AC}$ & 1 \\
\hline 644 & 14.7875 & -72.4389 & SMC-DEM101 & 0.010 & 6.7 & 0.15 & NA & 1 \\
\hline 650 & 14.8083 & -72.4050 & SMC-DEM100,MA1128 & 0.010 & 7.2 & 0.20 & NA & 1 \\
\hline 651 & 14.8167 & -72.2919 & SMC-DEM102,H-A44,B-OB15 & 0.033 & 6.7 & 0.13 & NA & 1 \\
\hline 652 & 14.8083 & -72.6081 & B96,SMC_OGLE117 & 0.008 & 8.3 & 0.08 & $\mathrm{C}$ & 1 \\
\hline 655 & 14.8542 & -72.7386 & BS92 & 0.016 & 6.9 & 0.08 & A & 1 \\
\hline 665 & 14.9083 & -72.7472 & B-OB14 & 0.058 & 6.9 & 0.08 & A & 1 \\
\hline 666 & 14.9125 & -72.7772 & $\mathrm{H}-\mathrm{A} 46$ & 0.037 & 7.3 & 0.08 & A & 1 \\
\hline 667 & 14.9500 & -72.3339 & IC1611,K40,L61,ESO29SC27 & 0.013 & 8.1 & 0.08 & $\mathrm{C}$ & 1 \\
\hline 668 & 14.9875 & -72.3733 & H86-186,SMC_OGLE119 & 0.005 & 8.1 & 0.08 & $\mathrm{C}$ & 1 \\
\hline
\end{tabular}


Table 2. continued.

\begin{tabular}{|c|c|c|c|c|c|c|c|c|}
\hline ID & $\begin{array}{c}\alpha(2000) \\
(\mathrm{deg})\end{array}$ & $\begin{array}{c}\delta(2000) \\
(\mathrm{deg})\end{array}$ & Name & $\begin{array}{c}\text { Radius } \\
\text { (deg) }\end{array}$ & $\begin{array}{c}\log (\text { Age }) \\
(y r)\end{array}$ & $E(V-I)$ & Type & Class \\
\hline 669 & 14.9625 & -72.7175 & BS95 & 0.011 & 7.2 & 0.08 & $\mathrm{~A}$ & 1 \\
\hline 671 & 15.0042 & -72.3689 & IC1612,K41,L62,ESO29SC28 & 0.010 & 8.0 & 0.08 & $\mathrm{C}$ & 1 \\
\hline 675 & 15.0542 & -72.4622 & H86-188,SMC_OGLE121 & 0.010 & 7.9 & 0.08 & $\mathrm{AC}$ & 1 \\
\hline 681 & 15.0958 & -72.1994 & BS100 & 0.027 & 6.6 & 0.15 & NA & 1 \\
\hline 684 & 15.1125 & -73.0867 & B99,SMC_OGLE122 & 0.006 & 8.3 & 0.08 & $\mathrm{C}$ & 1 \\
\hline 685 & 15.1375 & -72.2397 & H86-189,SMC_OGLE123 & 0.003 & 8.8 & 0.08 & $\mathrm{C}$ & 3 \\
\hline 686 & 15.1375 & -72.2586 & H86-190,SMC_OGLE230 & 0.002 & 7.3 & 0.08 & $\mathrm{C}$ & 1 \\
\hline 688 & 15.1417 & -72.3656 & K42,L63,SMC_OGLE124 & 0.004 & 7.7 & 0.08 & $\mathrm{C}$ & 1 \\
\hline 691 & 15.1958 & -72.9281 & SMC_OGLE125 & 0.003 & 8.2 & 0.10 & $\mathrm{CA}$ & 1 \\
\hline 694 & 15.2083 & -72.5031 & SMC-DEM114 & 0.167 & 6.7 & 0.15 & NA & 1 \\
\hline 697 & 15.2292 & -72.4956 & B-OB18 & 0.042 & 6.8 & 0.14 & AN & 2 \\
\hline 700 & 15.2417 & -72.5403 & H86-191,SMC_OGLE231 & 0.007 & 8.2 & 0.08 & $\mathrm{C}$ & 1 \\
\hline 701 & 15.2583 & -72.7514 & L65,H86-192,SMC_OGLE126 & 0.009 & 8.0 & 0.08 & $\mathrm{C}$ & 1 \\
\hline 705 & 15.3083 & -72.5508 & H86-194,SMC_OGLE232 & 0.004 & 8.4 & 0.08 & $\mathrm{C}$ & 1 \\
\hline 707 & 15.3250 & -72.2283 & H86-193,SMC_OGLE127 & 0.005 & 8.3 & 0.08 & $\mathrm{C}$ & 1 \\
\hline 714 & 15.4042 & -72.4069 & B105,SMC_OGLE128 & 0.003 & 7.3 & 0.08 & $\mathrm{C}$ & 1 \\
\hline 715 & 15.4125 & -72.4842 & H86-195 & 0.012 & 7.4 & 0.14 & A & 3 \\
\hline 717 & 15.4375 & -72.5644 & L66,SMC_OGLE129 & 0.009 & 7.3 & 0.12 & $\mathrm{C}$ & 1 \\
\hline 718 & 15.4375 & -72.9478 & B-OB20 & 0.033 & 6.8 & 0.20 & A & 1 \\
\hline 719 & 15.4667 & -72.1828 & B108,SMC_OGLE130 & 0.003 & 8.8 & 0.08 & $\mathrm{C}$ & 3 \\
\hline 722 & 15.5125 & -72.2106 & B-OB19 & 0.100 & 6.7 & 0.12 & A & 1 \\
\hline 723 & 15.5125 & -72.6739 & B-OB21 & 0.033 & 7.0 & 0.14 & A & 1 \\
\hline 724 & 15.5208 & -72.5992 & H-A48 & 0.023 & 6.7 & 0.18 & A & 3 \\
\hline 727 & 15.5125 & -72.5219 & SMC_OGLE131 & 0.006 & 8.2 & 0.08 & $\mathrm{CA}$ & 1 \\
\hline 729 & 15.5542 & -72.9664 & SMC_OGLE132 & 0.003 & 8.6 & 0.30 & $\mathrm{C}$ & 1 \\
\hline 735 & 15.6250 & -72.6281 & BS105 & 0.009 & 7.3 & 0.08 & $\mathrm{CA}$ & 1 \\
\hline 736 & 15.6292 & -72.3183 & SMC_OGLE133 & 0.003 & 8.8 & 0.08 & $\mathrm{C}$ & 1 \\
\hline 739 & 15.6583 & -72.6917 & BS274 & 0.016 & 7.0 & 0.10 & A & 1 \\
\hline 743 & 15.7125 & -72.4131 & SMC-DEM118,H-A50 & 0.025 & 6.9 & 0.14 & NA & 2 \\
\hline 745 & 15.7208 & -72.4147 & B114,SMC_OGLE234 & 0.007 & 7.1 & 0.12 & $\mathrm{NC}$ & 2 \\
\hline 750 & 15.7792 & -72.1072 & SMC-N76B,SMC-DEM120,MA1361 & 0.005 & 7.3 & 0.08 & $\mathrm{NC}$ & 1 \\
\hline 751 & 15.7750 & -72.4317 & B-OB23 & 0.040 & 6.9 & 0.14 & AN & 1 \\
\hline 752 & 15.7708 & -72.7008 & H-A51 & 0.017 & 7.3 & 0.08 & A & 1 \\
\hline 753 & 15.8000 & -72.2725 & K47,L70,SMC_OGLE134 & 0.006 & 7.3 & 0.08 & $\mathrm{C}$ & 1 \\
\hline 754 & 15.8208 & -72.7408 & SMC_OGLE135 & 0.006 & 8.7 & 0.08 & $\mathrm{AC}$ & 1 \\
\hline 756 & 15.8208 & -72.6158 & H-A52 & 0.047 & 7.1 & 0.15 & A & 2 \\
\hline 757 & 15.8417 & -72.4658 & SMC_OGLE136 & 0.007 & 8.1 & 0.08 & $\mathrm{CA}$ & 1 \\
\hline 758 & 15.8458 & -72.6517 & B115,SMC_OGLE137 & 0.007 & 7.1 & 0.13 & $\mathrm{C}$ & 1 \\
\hline 759 & 15.8625 & -72.0817 & H-A53 & 0.075 & 6.7 & 0.10 & AN & 2 \\
\hline 761 & 15.8708 & -71.9333 & B-OB24 & 0.070 & 7.0 & 0.08 & NA & 2 \\
\hline 762 & 15.8708 & -72.0567 & NGC371,SMC-N76,K48,L71 & 0.035 & 6.7 & 0.10 & NA & 1 \\
\hline 767 & 15.9542 & -72.0647 & SMC-N76A,L61-420,MA1411 & 0.003 & 8.8 & 0.08 & $\mathrm{NC}$ & 3 \\
\hline 769 & 15.9708 & -72.1031 & SMC_OGLE138 & 0.005 & 7.2 & 0.04 & $\mathrm{CA}$ & 2 \\
\hline 770 & 15.9708 & -72.1397 & SMC-N76C,L61-422,MA1423 & 0.003 & 8.3 & 0.08 & $\mathrm{NC}$ & 2 \\
\hline 771 & 15.9708 & -72.8261 & NGC376,K49,L72,ESO29SC29 & 0.015 & 7.2 & 0.08 & $\mathrm{C}$ & 1 \\
\hline 772 & 15.9833 & -72.6858 & SMC-DEM122 & 0.012 & 7.4 & 0.08 & NA & 3 \\
\hline 773 & 15.9958 & -72.8050 & BS114,SMC_OGLE235 & 0.058 & 7.1 & 0.08 & $\mathrm{AC}$ & 3 \\
\hline 776 & 16.0208 & -72.1208 & SMC_OGLE144,SMC_OGLE236 & 0.005 & 7.6 & 0.10 & CA 1 & \\
\hline 779 & 16.0292 & -72.0217 & BS117 & 0.014 & 7.3 & 0.08 & NA & 3 \\
\hline 780 & 16.0583 & -72.6469 & BS118,SMC_OGLE140 & 0.010 & 6.9 & 0.08 & A & 1 \\
\hline 781 & 16.0208 & -72.7181 & H-A55 & 0.043 & 6.8 & 0.13 & A & 2 \\
\hline 786 & 16.0708 & -72.3958 & SMC-DEM125 & 0.092 & 6.8 & 0.14 & NA & 2 \\
\hline 789 & 16.0833 & -72.0489 & SMC-DEM124 & 0.158 & 6.6 & 0.12 & NA & 2 \\
\hline 790 & 16.0917 & -72.8478 & BS121,SMC_OGLE237 & 0.013 & 7.9 & 0.04 & $\mathrm{C}$ & 3 \\
\hline 794 & 16.1167 & -72.1850 & BS123 & 0.009 & 7.5 & 0.08 & $\mathrm{CA}$ & 1 \\
\hline 795 & 16.1250 & -72.6192 & B121,SMC_OGLE141 & 0.005 & 8.0 & 0.08 & $\mathrm{C}$ & 1 \\
\hline 797 & 16.1250 & -72.5767 & BS124 & 0.013 & 7.3 & 0.08 & A & 1 \\
\hline 799 & 16.1500 & -72.1606 & K50,L74,ESO51SC15 & 0.008 & 6.9 & 0.14 & $\mathrm{C}$ & 2 \\
\hline 800 & 16.1667 & -72.5500 & BS125,SMC_OGLE143 & 0.013 & 7.5 & 0.20 & A & 1 \\
\hline 801 & 16.1583 & -72.6767 & B-OB25 & 0.033 & 7.0 & 0.14 & A & 2 \\
\hline 802 & 16.1625 & -72.8161 & H-A56,B-OB27 & 0.068 & 6.7 & 0.14 & A & 1 \\
\hline 803 & 16.1875 & -72.1328 & H-A54 & 0.050 & 6.9 & 0.13 & A & 1 \\
\hline 807 & 16.2417 & -72.4031 & B-OB28nw & 0.050 & 6.8 & 0.12 & AN & 1 \\
\hline
\end{tabular}


E. Chiosi et al.: Cluster and field SFR in the SMC, Online Material p 9

Table 2. continued.

\begin{tabular}{ccclccccc}
\hline \hline ID & $\begin{array}{c}\alpha(2000) \\
(\mathrm{deg})\end{array}$ & $\begin{array}{c}\delta(2000) \\
(\mathrm{deg})\end{array}$ & Name & $\begin{array}{c}\text { Radius } \\
(\mathrm{deg})\end{array}$ & $\begin{array}{c}\text { Log(Age }) \\
(\mathrm{yr})\end{array}$ & $E(V-I)$ & Type & Class \\
\hline 813 & 16.2792 & -71.9936 & NGC395,K51,L75,ESO51SC16 & 0.013 & 7.2 & 0.08 & NA & 1 \\
816 & 16.2958 & -71.9744 & SMC-N78D,SMC-DEM127 & 0.007 & 7.2 & 0.08 & NA & 1 \\
818 & 16.3042 & -71.9950 & SMC_OGLE146 & 0.004 & 7.3 & 0.08 & NA & 2 \\
819 & 16.3292 & -72.0033 & SMC-DEM126 & 0.032 & 6.9 & 0.14 & NA & 2 \\
820 & 16.3417 & -72.0431 & IC1624,K52,L76,ESO51SC17 & 0.007 & 8.3 & 0.08 & C & 2 \\
821 & 16.3375 & -72.1456 & SMC-DEM128 & 0.013 & 7.3 & 0.08 & NA & 2 \\
822 & 16.3458 & -72.0222 & SMC-N78,H-A57,B-OB26 & 0.050 & 7.0 & 0.12 & NA & 1 \\
825 & 16.4042 & -72.4658 & B-OB28se & 0.028 & 6.9 & 0.10 & AN & 3 \\
827 & 16.4208 & -72.0594 & SMC-N78C,SMC-DEM130,MA1543 & 0.006 & 7.2 & 0.20 & NA & 1 \\
828 & 16.4583 & -71.9511 & B128 & 0.006 & 8.2 & 0.08 & C & 1 \\
830 & 16.5000 & -72.3414 & BS131,SMC_OGLE150 & 0.005 & 8.1 & 0.08 & C & 1 \\
831 & 16.4875 & -72.5364 & BS129 & 0.015 & 6.9 & 0.14 & AN & 2 \\
835 & 16.5542 & -72.7942 & SMC_OGLE151 & 0.010 & 8.1 & 0.10 & AC & 1 \\
837 & 16.5583 & -72.2536 & B-OB29 & 0.083 & 6.9 & 0.10 & A & 2 \\
847 & 16.7000 & -72.2736 & K54,L79,ESO29SC31 & 0.007 & 8.0 & 0.04 & C & 3 \\
850 & 16.7375 & -72.8472 & SMC-DEM133 & 0.010 & 7.7 & 0.15 & NA & 3 \\
853 & 16.7667 & -72.2436 & BS137 & 0.012 & 7.1 & 0.12 & A & 1 \\
854 & 16.7583 & -72.6217 & B129,SMC_OGLE154 & 0.009 & 8.2 & 0.14 & CA & 1 \\
858 & 16.8250 & -72.5892 & B130 & 0.010 & 7.3 & 0.08 & A & 1 \\
860 & 16.8667 & -72.4933 & K56,SMC_OGLE155 & 0.009 & 8.2 & 0.14 & C & 2 \\
861 & 16.8667 & -72.7694 & L80,SMC_OGLE156 & 0.010 & 8.1 & 0.08 & C & 1 \\
864 & 16.8833 & -73.1197 & K55,L81,SMC_OGLE157 & 0.008 & 8.7 & 0.15 & C & 1 \\
865 & 16.9167 & -72.2694 & B-OB30 & 0.033 & 6.8 & 0.10 & A & 1 \\
866 & 16.9167 & -72.5667 & H-A58 & 0.042 & 7.0 & 0.10 & A & 1 \\
876 & 17.0792 & -72.8842 & NGC419,K58,L85,ESO29SC33 & 0.023 & 8.6 & 0.08 & C & 3 \\
881 & 17.1542 & -72.4392 & SMC_OGLE160 & 0.005 & 7.6 & 0.08 & CA & 2 \\
888 & 17.2625 & -73.0867 & K61,SMC_OGLE161 & 0.008 & 8.0 & 0.08 & C & 1 \\
\hline & & & & & & & &
\end{tabular}

